\title{
La investigación en Psicología Diferencial cognoscitiva: Estrategias de Primera Generación
}

\author{
M. DE JUAN-ESPINOSA \\ R. COLOM \\ Universidad Autónoma de Madrid
}

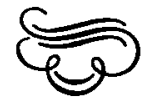

Resumen

En el presente artículo se tratan de enmarcar las tradiciones de investigación en psicologia diferencial, a través de una clasificación de estrategias de investigación. Estas estrategias de investigación cubrirán el espectro empirico de la psicología diferencial cognoscitiva. Se analizan las que hemos dado en denominar Estrategias de Primera Generación: estrategias de correlatos, estrategias heurísticas (estrategia de simulación matemática), y estrategias de comparación de grupos. La caracteristica más sobresaliente de estas estrategias de primera generación es que su rango de aplicación está limitado a actividades cognoscitivas especificas. Este análisis de las estrategias de primera generación conecta con las guias teóricas expuestas por los autores en otro lugar.

\section{Abstract}

This paper attempts to present the research traditions in differential psychology, through a taxonomy of research strategies. Such strategies will cover the empirical range of Cognitive Differential Psychology. First Generation Strategies are analyzed, including correlate strategies, beuristic strategies (rational strategy, computer simulation strategy, and mathematical simulation strategy), and group comparison strategies. These first generation strategies most important characteristic is that their domain of application is restricted to specific cognitive activities. This study/connects with the theoretical guides exposed by authors elsewhere.

Dirección de los autores: Universidad Autónoma de Madrid. Facultad de Psicología. Dpto. de Psicología Biológica y de la Salud. Canto Blaco, 28049 Madrid. 


\section{INTRODUCCIÓN}

Frecuentemente, en la literatura científica, se establece un fuerte paralelismo entre Psicología Diferencial-Psicología Correlacional, y Psicología Diferencial-Psicología Psicométrica, de tal forma que dichos términos suelen usarse de forma intercambiable (Cronbach, 1957; Cronbach, 1975; Hunt y MacLeod, 1979; Sternberg, 1981). Dicho paralelismo ha ido más allá de lo que hubiera sido de desear y, paulatinamente, se ha ido convirtiendo en lo que llamaríamos una «identidad conceptual tácitamente asumida». El resultado de dicha identidad ha sido doble. Por un lado, un enorme desarrollo del enfoque psicométrico de las diferencias individuale, o Psicología Diferencial Psicométrica, cuya orientación al objeto de estudio se ha dirigido casi exclusivamente al estudio de las diferencias inter-individuales o inter-grupales dejando de lado uno de los focos de estudio más importantes: la variabilidad intra-individual; variabilidad intra-individual que puede permitir el avance en el conocimiento de los mecanismos que se supone describen y/o explican la conducta (Tous, 1986). Esta falta de atención a las diferencias intra-individuales, restringe de facto el campo de conocimiento. Por otro lado, tradicionalmente se ha asignado al estudio de las diferencias humanas una vertiente metodológica fundamentalmente correlacional. Tanto desde el punto de vista histórico, como desde el carácter marcadamente aplicado de esta disciplina, esta última identidad parece justificada. En el presente artículo nos ocuparemos fundamentalmente de este último paralelismo y de sus posibles alternativas bajo el marco de lo que llamaremos el enfoque cognoscitivo de la Psicología Diferencial, o Psicología Diferencial Cognoscitiva.

Desde la perspectiva histórica, fueron tres los motivos que consiguieron dotar al London University Collegue de una tradición en la aplicación de la metodología correlacional al estudio de las diferencias humanas. En primer lugar, los trabajos sobre relaciones entre variables del pionero de la Psicología Diferencial, Francis Galton (1822-1911), que le llevaron a idear el llamado índice de co-relación (1), y a publicar su artículo "Co-relaciones y su medición a partir, especialmente, de datos antropométricos». En segundo lugar, el perfeccionamiento de esta idea, llevado a cabo por su discípulo Karl Pearson, así como la base matemática con que dotó al método estadístico de Galton (Sahakian, 1975). Y, por último, la formulación del Análisis de Factores por Charles Spearman (1863-1945). Esta tradición se extendió inmediatamente a toda la geografía de la investigación psicológica.

Desde la perspectiva aplicada, como afirman Forteza y Prieto (1981) «(...) el objeto de la Psicología Diferencial no ha sido nunca la conducta en abstracto, sino siempre abordada en el contexto de un marco aplicado y concreto, lo más próxima posible a sus manifestaciones en la vida real y rehuyendo, tajantemente, los estrechos márgenes de una ciencia ceñida a experimentos de laboratorio" (p. 68). Este carácter marcadamente aplicado, junto al gran avance de la metodología correlacional, ha conducido al desarrollo de un modelo de psicodiagnóstico diferencial basado fundamentalmente en pruebas psicométricas que ha sido, y sigue siendo, predominante en la psicología aplicada. Pero, por otra parte, ha convertido el estudio de las diferencias humanas en una tecnología más que en una disciplina científica teóricamente fundamentada (Caparros, 1982). 
De lo dicho podría pensanse que la Psicología Diferencial Psicométrica ha carecido de teorías sustentadoras. Sin embargo, esto no es cierto. Desde los comienzos han existido formulaciones teóricas explícitas elaboradas desde los primeros estudios de F. Galton, C. Spearman, etc., hasta los psicólogos diferenciales de la actualidad como R. B. Catell, H. J. Eysenck, etc... Olvidar este hecho sería un grave handicap para el desarrollo de teorías sobre las diferencias humanas, $\mathrm{e}$ incluso para las teorías psicológicas en general. no obstante, dichas formulaciones; basadas en "macro-hipótesis», se han caracterizado por una carencia notable de un entramado teórico de cadenas de hipótesis especificas que facilitasen su falsabilidad.

\section{EL MÉTODO DE ESTUDIO DE LAS DIFERENCIAS HUMANAS}

De lo anteriormente mencionado se desprende una evidente estructura lógica interna que adscribe una metodología determinada, la correlacional, a un entramado teórico diferencial sustentado por una tecnología de carácter psicométrico. Sin embago, la asociación de un modelo metodológico específico a un marco teórico, tiene una serie de problemas asociados, que exigen al investigador una mayor flexibilidad. Esta exigencia se incrementa cuando el ámbito de estudio corresponde al de las ciencias sociales, donde el objeto de conocimiento lo constituyen sistemas abiertos (Bertalanffy, 1974), y el nivel explicativo se haya impregnado de una caracterización funcionalista y teleológica (Fodor, 1968; Pinillos, 1981; Taylor, 1979), más allá del reduccionismo explicativo de relaciones causales (Bowers, 1973), y cuya contrastabilidad se encuentra dificultada por los problemas derivados de la llamada Teoría de los dos sujetos experimentales (Seoane, 1981).

Podría parecer que la correspondencia Método-Teórica es algo muy de desear, y esto es en realidad lo que confiere hoy en día, desde la academia, a cualquier rama de la psicología, el carácter de disciplina científica. No obstante, esta correspondencia aparentemente deseable, no es más que un resabio del Positivismo Lógico (Brown, 1977) que impregna el Hiperpositivismo reinante en la psicología actual (Zaccagnini, comunicación personal). Hacer depender la cientificidad de los supuestos teóricos de una metodología determinada, además de conducirnos a aceptar ingenuamente la existencia de un modelo in aeternum (Pinillos, 1981) puede llevar, y ha llevado, a una peligrosa relación de dependencia del objeto de conocimiento de una metodología específica, cuyos resultados pueden ser la reducción de sus contenidos y de su aspecto de alcance, relevantes para dicha teoría. Es la metodología la que ha de adaptarse, mediante adecuadas estrategias de investigación, a los objetivos de la teoría y no a la inversa, situación que podríamos formular como Teoria ancille Metodologiae. En resumen, frente a una concepción de la teoría como constructo intelectual sin significado hasta que se lo concreta mediante observaciones (Feigl, 1970), parece más correcto sostener que son las teorías las que dan significado a nuestras observaciones (Rusell Hanson, 1958; Brown, 1977).

Siendo las diferencias individuales uno de los problemas más recalcitrantes (2) en la investigación psicológica que urge un análisis explicativo, cabría preguntarse por la metodología apropiada para hacer frente al mismo. A juzgar por el artículo seminal de Cronbach (1957) sobre «Las dos 
disciplinas de la Psicología Científica», y la tradición investigadora imperante hasta finales de los sesenta, principios de los setenta, la respuesta estaría en la metodología correlacional, No obstante, la llamada a la reunificación de las dos disciplinas - Correlacional y Experimental - tuvo dos repercusiones importantes. Por un lado, el reconocimiento de la necesidad de incorporar el estudio de las diferencias individuales a los trabajos experimentales; y por otro, la urgencia de elaborar un marco teórico comprehensivo y coherente capaz de guiar investigaciones más moleculares sobre las diferencias humanas.

El Simposium sobre «Aprendizaje y Diferencias Individuales», presidido por Robert M. Gagne, y celebrado en la Universidad de Pittsburgh en abril de 1965, fue crucial para introducir nuevas formas y estrategias de trabajo en el estudio de las diferencias humanas. Conferencias como la de Edwin A. Fleischman sobre "Diferencias Individuales y Aprendizaje Motor"; o la de Paul M. Kjeldergaard sobre «Simulación de la Cognición y el Aprendizaje» y su réplica por parte de Lee W. Gregg «Modelos de Simulación y Diferencias Humanas»; etc., por citar algunas de entre la gran cantidad y diversidad de conferencias y trabajos, contribuyeron a que se tomase conciencia de la necesidad, y posibilidad de adoptar nuevas formas de investigación, para dar respuesta a nuevos problemas enfocados hacia explicaciones más moleculares y teóricamente basadas sobre las diferencias humanas.

La corta historia, poco más de veinte años, que ha seguido al mencionado Simposium, se ha visto plagado de un resurgir del estudio de las diferencias individuales en el plano general, y de la aparición y paulatina consolidación de una "nueva" perspectiva de la Psicología Diferencial. Temas como la importancia de la velocidad de procesamiento, estrategias de resolución de problemas, base de conocimientos, procesos de control ejecutivo, representación mental, y un largo etcétera, para la comprensión de las diferencias humanas, marcaron un estilo distinto de investigar y comenzaron a aparecer, tímidamente en un principio, en revistas tan prestigiosas como las cuatro ediciones del Journal of Experimental Psychology, Memory and Cognition, Psychological Review, etc., y sobre todo en una nueva revista Intelligence; posteriormente se han ido pasando a una mayor consolidación de tal forma que autores como Pellegrino y Glaser (1979) con su artículo "Correlatos cognitivos y componentes en el análisis de las Diferencias Individuales"; Sternberg (1981) con otro artículo en el cual ampliaba la taxonomía de estrategias de investigación de los mencionados autores, o el libro de Cole y Menas (1981) «Estudios comparativos de cómo piensa la gente» (3), han marcado un cambio profundo en la manera de trabajar sobre las diferencias individuales en cognición.

En esta perspectiva, el nuevo foco de interés no se centraba tanto en conocer en qué y cuánto diferían los sujetos entre sí -tema tradicional del enfoque psicométrico de las diferencias humanas-, sino en cómo y por qué lo hacían. Es decir, interesaban no tanto los productos, sino los procesos diferenciadores que mediaban entre el input y el output. Junto a la búsqueda incansable de dimensiones diferenciales con un alto grado de estabilidad, aparecieron los intentos de modificación de las diferencias, de tal forma que algunos autores asumieron como objetivo explícito la búsqueda de "formas de mejorar, o al menos reducir, las diferencias» (Campione y Brown, 1979, p. 140). 
Para asumir plenamente este desafío, urgía un «nuevo» marco teórico que huyera de la circularidad de explicar las diferencias individuales a través de atributos o rasgos -medidos a su vez por tests psicométricos cuya validez dependía de su validez discriminante, construidos para medir las mencionadas diferencias-, y que puediera incorporar los logros sobre inteligencia, aptitudes, estilos cognitivos, etc., propios de la tradición diferencialista psicométrica, revitalizándolos con nuevos marcos explicativos y nuevas técnicas de investigación. Las teorías cognitivas y las derivadas del Procesamiento de la Información, al ofrecer un entramado de conceptos amplio que rompian las explicaciones ad hoc a través de variables intermediarias o constructos hipotéticos, mediante la afirmación de la existencia de todo un sistema cognitivo, y una riqueza y flexibilidad metodológica de carácter proposicional e informativo, han sido las candidatas más firmes hasta el momento.

Una primera forma de aproximación al estudio de las diferencias individuales en cognición suele comenzar con una metodología correlacional basada en modelos lineales brivariados o multivariados. De esta forma, sería factible contestar a la existencia de diferencias, así como a su generalidad, sobre un conjunto de variables, dejando a un lado los efectos de tratamientos diferenciales o la manipulación de condiciones. Como ya apuntara Sargent (1942), este tipo de aproximaciones, propias de diseños ex post facto, no nos permiten describir el método utilizado por un sujeto en la ejecución de una tarea, ni nos muestra los procesos cognitivos implicados en dicha tarea. En este sentido, la metodología factorial es la más empleada.

Los métodos experimentales y cuasiexperimentales tienen la ventaja de definir procesos, componentes, o parámetros procesuales, en términos de diferencias entre dos condiciones. Estas comparaciones nos permiten hacer inferencias acerca de dicho proceso en una determinada tarea. Lógicamente, dicha diferencia entre condiciones es un indicador de la validez aparente del parámetro en cuestión, siempre y cuando el proceso o parámetro haya sido definido razonablemente en términos de la mencionada diferencia (Baron y Treiman, 1980). Estos métodos se utilizan generalmente para hallar el llamado déficit diferencial, mediante una estrategia de comparación de grupos, o utilizando un grupo definido con dos tareas, una experimental y otra control. Dichos métodos, salvando algunos problemas que comentaresmos más adelante (Baron y Treiman, 1930; Chapman y Chapman, 1973, 1985; Loftus, 1978), permiten un acercamiento a los problemas de causación de la variedad, así como a la posibilidad de modificar dichas diferencias. Por otro lado, los métodos de simulación, así como los de Inteligencia Artificial, han ayudado a la comprensión de las diferencias humanas (Kjeldergaard, 1966; Greeg, 1966; Laughery y Gregg, 1962; Newell y Simon, 1972; Dhen y Schanck, 1972, Young, 1978). Evidentemente estos métodos no son excluyentes, sino que son distintas aproximaciones a un mismo problema (recalcitrante), a saber: la conceptualización y explicación del comportamiento diferencial de los individuos.

\section{HACIA UNA CLASIFICACIÓN DE ESTRATEGIAS DE INVESTIGACIÓN}

Pellegrino y Glaser (1979), y posteriormente Sternberg (1981), han realizado un intento de clasificación de los distintos tipos de estudios, o es- 
trategias de análisis de las diferencias individuales en cognición. De acuerdo a la primera clasificación (Pellegrino y Glaser, op. cit.) se establecen las aproximaciones de correlatos cognitivos (examen del rendimiento de los sujetos en la resolución de tareas que se supone miden las capacidades humanas básicas del PI, utilizándose diseños con sujetos de alta y baja capacidad, identificados mediante tests psicométricos de aptitud, y analizándose su rendimiento frente a tareas básicas del PI) y de componentes cognitivos (se analiza el rendimiento de los sujetos en tareas de los test psicométricos estandarizados de aptitudes de capacidades mentales, descomponiéndose las tareas hasta llegar a los componentes más sencillos de procesamiento que configuran el rendimiento en la tarea). Sternberg (op. cit.) amplía la lista con dos nuevas aportaciones: adiestramiento cognitivo (estudio de la capacidad de adiestramiento de los procesos cognitivos, como forma de verificar la importancia relativa de estos procesos en la ejecución de pruebas de capacidades mentales, siendo un objetivo importante demostrar la perdurabilidad del adiestramiento y la generabilidad de la destreza adiestrada) y contenidos cognitivos (análisis de las diferencias en los contenidos y estructuras de conocimiento, de sujetos de alta y baja capacidad en determinadas tareas, siendo el objetivo descubrir la diferencia y su naturaleza en la base de conocimientos de los sujetos, y la utilización que hacen éstos de tal base). Tal y como apuntan los mencionados autores, estas clasificaciones no pretenden ser exhaustivas, ni establecer un criterio de exclusión entre cada una de ellas. Es más la distinción resulta a veces imposible (Sternberg, 1981), y sólo tiene carácter didáctico.

Por nuestra parte, creemos que sería conveniente reformular en parte estas clasificaciones añadiéndoles algunos trabajos que, aun perteneciendo a otros campos más generales, como en el caso de las simulaciones, posibilitan vías de trabajo útiles en el estudio y análisis de las diferencias individuales.

En primer lugar, se podrían identificar tres estrategias primitivas, o de primera generación, de aproximación al análisis de las diferencias humanas. El concepto de primera generación hace referencia al estudio de ámbitos concretos de las diferencias humanas, sin tender a la creación de una teoría general e integrada sobre tales diferencias. La primera estrategia conceptualizaría las diferencias como puntos a lo largo de un continuo de escala, que vendrían a representar desviaciones respecto a una medida de tendencia central, tomadas en términos de dispersión. Bajo esta estrategia de investigación, se dice que el componente diferencial ha sido identificado, cuando los sujetos se ajustan a determinadas medidas o parámetros. Ejemplos de esta primera estrategia serían las estrategias de correlatos, tanto en el análisis de los correlatos biológicos, como en el estudio de los correlatos psicológicos. En segundo lugar, nos encontraríamos con las estrategias beuristicas — p.e. análisis racional de John B. Carrol (1976-1983)—, y con la justificación diferencial de los modelos - p.e. trabajos de simulación por ordenador del funcionamiento de las imágenes mentales de Stephen Kosslyn (1984), Kosslyn, Brown, Cave y Wallach (1984), o los trabajos sobre simulación matemática de resolución de problemas de Mumaw, Pellegrino y Glaser (1980)- . Por último, la tercera estrategia de investigación entendería las diferencias como representaciones de grupos identificados a priori. Se dice que el componente ha sido identificado, en la medida en que se in- 
fiere de la comparación de dichos grupos. Este punto de vista procede de la tradición en psicología clínica y pretende detectar el déficit diferencial - p.e. los trabajos del Grupo de Illinois (Brown, Campione, Ferrara, etc.).

En segundo lugar, nos encontraríamos con estrategias de investigación de amplio espectro, o metodologías de segunda generación. Según Rose (1980), las metologías de segunda generación se caracterizarían por incrementar el poder descriptivo y explicativo de las metodologías que hemos denominado de primera generación - de carácter más restringido y específico-, y aumentar el alcance de las posibles aplicaciones. Estas metodologías serían útiles para aumentar la capacidad de descubrimiento y análisis de los componentes de procesamiento. Entre las estrategias de segunda generación más firmemente candidatas se encontrarían: El Método de Generalización de Factores Aditivos de Calfee (1976); El Análisis Componencial de Robert Sternberg (1977), y El Análisis Factorial Confirmatorio de Fredeiriksen (1978).

En resumen, el objetivo general de estas estrategias es posibilitar la caracterización de las diferencias individuales en términos de estructuras y procesos hipotéticos que emanan de estudios teóricos y empíricos de la cognición humana y el desarrollo cognitivo. Esta aproximación enfatiza que el uso primordial de las medidas de inteligencia y aptitudes, como constructos diferenciales, no es predecir, sino indicar cómo puede mejorarse la ejecución intelectual. Esta meta puede lograrse en la medida en que las diferencias individuales puedan interpretarse en términos de componentes que posibilitan o dificultan la ejecución cognitiva (Pellegrino y Glaser, 1979).

En este trabajo sólo vamos a tratar las estrateagias de primera generación, dejando para una segunda entrega, el análisis y discusión de las estrategias de segunda generación, puesto que dada su importancia, el estudio pormenorizado y exhaustivo que requieren haría excesiva la longitud de este artículo.

\section{ESTRATEGIAS DE PRIMERA GENERACIÓN}

\section{Estrategias de correlatos}

El objetivo general de la estrategia de correlatos es establecer la relación diferencial entre medidas generales de constructos diferenciales -inteligencia, aptitud, etc.- y componentes diferenciales del PI; entre medidas generales de constructos diferenciales y componentes neuropsicológicos -Potenciales Evocados-, y entre componentes diferenciales del PI y componentes neuropsicológicos.

Dos grandes apartados se incluyen en el ámbito de esta estrategia de investigación. Por una parte, aquél que se centra en los correlatos biológicos, y por otra, aquél que focaliza su atención en los correlatos cognitivos. Ambos comparten un esquema de trabajo similar en cuanto al entramado fundamental.

\section{Estrategia de Correlatos Biológicos}

La estrategia de correlatos biológicos pretende analizar en qué medida los constructos diferenciales (actitud, capacidad, etc.) y/o los componentes 
diferenciales del PI se reflejan en la base biológica en la que se implementan. Es decir, dado el lenguaje molar de la Psicología Diferencial (psicométrica, y en menor medida cognoscitiva), realizar una descripción de cómo un lenguaje más molecular (el biológico) puede dar cuenta de los mencionados constructos y componentes, además de servir de límite y criterio de verosimiltud en las elaboraciones teóricas de los psicólogos (Anderson, 1976).

En una primera aproximación se podría hablar de un discurso psicológico (P), y un discurso neuropsicológico (NP), siguiento a FarrelL (1983). Para este autor, el discurso NP trataría de características neuroelectroquímicas - p.e. eventos del sistema nervioso durante un determinado intervalo-. Por su parte, el discurso $P$ trataría del papel jugado por este conjunto de características en la actividad adaptativa del organismo.

Gale y Edwars (1983) nos advierten de un problema en el estudio de los correlatos biológicos que se patentiza en el principio de la integración funcional. Según este principio, una unidad funcional está implicada en varios sistemas funcionales, cada uno de los cuales tiene propiedades funcionales individuales y generales. Esto impide una localización de funciones, haciendo necesaria una distinción entre condiciones necesarias y suficientes de una función - p.e. una lesión puede producir una alteración conductual, pero sólo se puede decir que las partes dañadas del cerebro son necesarias, y no necesariamente suficientes, para la ejecución de la función correspondiente-. Además, diferentes funciones psicológicas, observadas como diferentes conductas, pueden estar representadas en el SN por uno o varios sistemas neurofisiológicos, y viceversa.

En términos generales, se encuentran en la literatura, básicamente, dos acepciones de correlato biológico (Farrrel 1983):

1. Buscar condiciones corporales de las que depende la conducta adaptativa, esperando encontrar condiciones de dependencia necesarias. Bajo esta acepción se pretenden descubrir conexiones causales entre los antecedentes (neurofisológicos) y los consecuentes (conductuales).

2. Cuando investigamos un evento, echando mano de los discursos $P$ y NP, estudiamos, por una parte, cómo ciertas partes corporales, estados, y eventos, median ciertos estados psicológicos, eventos, y actividades, y por otra, que los últimos son idénticos a los primeros. Es decir, los potenciales de espiga no causan la transmisión de información, sino que son esa transmisión (relación de identidad).

¿Qué importancia tienen este tipo de estudios para la Psicología Diferencial Cogniscitiva? Como dicen Gale y Edwards (op. cit.) cada organismo es un caso único. Parece lógico afirmar que las diferencias individuales en el sistema nervioso (Eysenck, 1985) interfuncionan * con las características ambientales para producir un patrón experimental único. Y es precisamente un patrón de este tipo el que interesa a la psicología diferencial cognoscitiva. Pero no en sí, sino en cuanto a cómo se produce esa interfunción entre los sistemas nerviosos diferentes (base biológica) y esas características ambientales, que, a través de la acción, van conformado al individuo, que no es nada más ni menos que un acúmulo organizado en modelos (Zaccagnini, 1985), de patrones experienciales producto de la mencionada interfunción. 
Las fases generales del procedimiento de investigación en estrategias de correlatos biológicos son:

1. Adopción de un modelo de funcionamiento neurológico diferencial. Por ejemplo, aquellos que se derivan de la diferenciación hemisféricocerebral y que han sido recogidos en el modelo de memoria distribuida de Hunt (1971).

2. Se hipotetiza la existencia de una relación entre uno o varios parámetros o medidas del modelo neurológico, y medidas de constructos diferenciales, como son los tests psicométricos; y/o medidas de parámetros cognitivos, como por ejemplo, la velocidad de acceso léxico.

3. Se evaluan a los sujetos en los mencionados constructos diferenciales, y se establecen grupos de rendimiento diferencial en los tests empleados para medirlo. A continuación se evalúa a estos sujetos bajo condiciones de laboratorio en medidas neurológicas como, por ejemplo, en la velocidad de aparición del potencial P-300.

4. Aplicando técnicas estadísticas bivariadas o multivariadas, se observan las relaciones halladas y se deduce la consistencia de la relación covariante hipotetizada.

Durante los últimos años se ha trabajado con potenciales evocados, tales como la VNC (variación negativa contingente) y con el PEP (potencial Evocado Promediado). Existe poca o nula evidencia de relación entre VNC e inteligencia - parece estar relacionado con parámetros atencionales-. Los PEP - potenciales positivos o negativos- se normalizan después de 500 ó $1.000 \mathrm{msg}$. Su relación con el constructo inteligencia se analiza durante los 250 primeros msg. La morfología de la onda varía de individuo a individuo. Esta varariación está en función de la naturaleza del estímulo, de la localización de los electrodos, de la intensidad estimular, etc. Chalke y Ertl (1965) señalaron que las latencias visuales PEP cortas correlacionaban con un CI elevado. Y en general, se sabe que el CI alto está relacionado significativamente con latencias cortas, amplitudes grandes y baja variabilidad (Eysenck, 1985).

Eysenck (op. cit.) presenta tres paradigmas que han encontrado correlaciones elevadas entre potenciales evocados y CI, a saber: el paradigma de Hendrickson (1982), el paradigma de Schafer (1982); y el paradigma de Robinson (1982). No obstante, nosotros no nos detendremos en una revisión de estos paradigmas. Sin embargo, mencionaremos las tres variables que postula el modelo de Eysenck-Turneaux (1982) en las que se dividiria la inteligencia: velocidad, persistencia y revisión del error.

Por último, determinados autores, como por ejemplo Jolhn Boddy (1978), identifican los componentes secundarios de los potenciales evocados con la percepción consciente. No obstante, para Farrel (1983), sólo se puede afirmar que estos componentes secundarios de los PE son manifestaciones de complejos estados neurales subyacentes al fenómeno psicológico de la percepción consciente.

Estos estudios, en cierta medida, y depende para quién, esperanzadores, se encuentran con un grave handicap al tratar con individuos humanos: coordinar la complejidad fisiológica, la variabilidad de la conducta humana, y la capacidad lingüistica que sirve de transmisor de experiencias. En nuestra opinión este handicap se vería disminuido, a través de una par- 
ticipación conjunta de los estudiosos del discurso P, y del discurso NP. La Psicología Diferencial Cognoscitiva tiende, en su espíritu, a ese tipo de congregaciones. Como afirman Tzeng y Hung (1985) «Los investigadores deben enfrentarse a las diferencias individuales y realizar serios intentos para dar cuenta de la varianza resultante. Este tipo de conocimiento sofistizará nuestras teorías y refinará nuestras metodologías. Entonces, y sólo entonces, podremos empezar a esperar una teoría unificada de la ciencia neurocognitiva». Nosotros nos ascribimos a esta postura.

\section{Estrategia de Correlatos Cognitivos.}

En los estudios correlacionales se toman dos o más medidas que suceden de forma natural, y se establecen inferencias al advertir cómo co-varían las medidas. Según Hunt (1985) determinados fenómenos sólo pueden investigarse con este tipo de estudios correlacionales. Por ejemplo, no conocemos la forma de manipular la rapidez de acceso léxico de un individuo, por lo que no se puede estudiar experimentalmente.

El gran problema que se encuentran los estudios correlacionales es la interpretación de los resultados. Para Hunt (op. cit.) el problema se resuelve desde el momento en que el estudio se realiza en el contexto de una teoría.

La estrategia de correlatos cognitivos pretende descubrir una relación entre dos variables teóricas (p.e. inteligencia verbal y aptitud innémica).

Para ello deben estudiarse las relaciones entre variables observables y las variables teóricas de interés, y las relaciones entre las variables teóricas per se. Este tipo de estudios exigen poseer un modelo explícito sobre cómo interactúan las variables teóricas para producir la ejecución observable.

Esta estrategia de investigación trata de especificar los componentes diferenciales del PI que están relacionados diferencialmente con niveles altos y bajos de puntuación en medidas de una aptitud intelectual. Su procedimiento general es el siguiente:

A. Adopción o diseño de un modelo de procesamiento de información relevante al fenómeno que se pretende analizar. Por ejemplo, Hunt, Frost y Lunneborg (1973) construye el Modelo de Memoria Distribuida en el que se van a basar posteriormente, para explicar la relación entre velocidad de acceso léxico y capacidad verbal.

B. Se hipotetiza la existencia de una relación entre uno o varios componentes del procesamiento de la información y medidas de constructos diferenciales de carácter psicométrico - por ejemplo, velocidad de acceso léxico y medidas de aptitud verbal-.

C. Se evalúa a los sujetos en los mencionados constructos mediante tests psicométericos empíricamente derivados - por ejemplo; test de Inteligencia General o aptitudes como la verbal-.

D. Se establecen grupos de alto o bajo nivel de puntuación en los tests aludidos, y se evalúa a los sujetos elegidos bajo condiciones de laboratorio, en tareas de PI definidas por investigaciones experimentales previas - por ejemplo; emparejamiento de letras (Posner, Boies, Eichelman y Taylor, 1969); capacidad de memoria a corto plazo (Peterson y Peterson 1959); examen de memoria a corto plazo (Stndenberg, 1969); etc.- .

E. Aplicando técnicas estadísticas bivariadas — correlación simple (p.e. 
Cohen Y Standenberg, 1977)—o multivariadas —análisis factorial (p.e. Hunt, Frost y Lunneborg, 1973); correlación canónica (p.e. Lunneborg, 1977); etc.- se observan las relaciones halladas, y se deduce la consistencia de la elación covariante hipotetizada.

Este tipo de estudios surgió ante la necesidad de ofrecer un marco teórico científico al estudio de constructos diferenciales como el de Aptitud Verbal (Hunt y Lansman, 1975). La Psicología Diferencial Psicométrica, centra sus propósitos en la clasificación de aquellos aspectos en los que difieren los sujetos. A al fin se plantean un conjunto de constructos hipotéticos o variables mediadoras medidas a través de tests psicométricos que hipotéticamente se sitúan en un plano explicativo de los productos diferenciales de los individuos. La Psicología Diferencial Cognoscitiva, sin desdenar la utilidad científica de las clasificaciones, de la que es deudora, pretende describir los procesos mediante los cuales un sujeto, o diferentes sujetos, se enfrentan a un mismo problema.

Desde el punto de vista de la estrategia de investigación basada en correlatos, el objetivo estaría en la estimación de parámetros o características cuantitativas que representan la duración o nivel de realización de uno o varios componentes del procesamiento de información en la realización de tareas que los implican, y analizar en qué medidas dichos componentes correlacionan entre sí, a través de los individuos, y con las puntuaciones en medidas que se supone evalúan constructos diferenciales. Es decir, busca especificar las capacidades básicas del procesamiento de la información que están relacionadas diferencialmente con distintos niveles de aptitud o inteligencia (Pellegrino y Glaser, 1979).

En resumen, la ausencia de una urdimbre teórica de carácter molecular en la Psicología Diferencial Psicométrica, podría quedar reflejada en el siguiente comentario de Royce: «En pocas palabras, me adscribo a la estrategia de permitir que sean los datos los que guíen nuestras conceptualizaciones, y no a la inversa» (1973, p. 391)

Por lo tanto, en lugar de intentar elaborar conclusiones teóricas a partir de puntuaciones correlacionadas de un test derivado empíricamente, con otro test dé estas características - tal como se hace y se sigue haciendo en la Psicología Diferencial Psicométrica-, se pretende elaborar conclusiones teóricas de las puntuaciones correlacionadas de un test empíricamente derivado con parámetros generados por un modelo cognitivo de algún aspecto del funcionamiento mental - por ejemplo, el Modelo de Memoria Distribuida (Hunt, 1971; Hunt, Frost y Lunneborg, 1973).

\section{Estrategias heurísticas}

En este tipo de estrategias de investigación se pretende fundamentalmente, identificar aquellos componentes del procesamiento de la información que son responsables en la ejecución de una tarea y que, por tanto, dan cuenta de los diferentes niveles de realización de la misma. La estrategia de correlatos intenta unir paradigmas y tareas experimentales usados en la investigación cognitiva con las cualificaciones de los tests psicométricos, mientras que las estrategias heurísticas, a través de un análisis racional y empírico de las demandas de procesamiento en una tarea específica comprendida, por lo regular, en los tests psicométricos, y derivando un modelo de 
ejecución de tales tareas, pretende investigar las fuentes de las diferencias individuales que contribuyen a la evaluación de constructos diferenciales y poner a prueba los items característicos. Es decir, se enfatiza el análisis de tareas para la identificación de componentes cognitivos en la ejecución de test a los cuales pertenecen dichas tareas.

El tipo de trabajos va desde un análisis extensivo de muchos tests, hasta un análisis intensivo en el que se elaboran modelos más específicos y detallados para la ejecución de un único tipo de item.

Los tipos de estrategias oscilan, de igual manera, entre el análisis racional (Carroll, 1976, 1979, 1983; Elshout, 1978; Snow, 1980; Stankov, 1980); la simulación por ordenador (Kjerdergaard, 1966; Kotovsky y Simon, 1973; Simon y Kotovsky, 1963); simulación matemática o análisis experimental de modelos de procesos (Pellegrino y Glaser, 1980; Stenberg, 1977; etc.) y diversas combinaciones de las diferentes estrategias mencionadas.

\section{Estrategia racional}

Siguiendo la línea de urgencia teórica para la interpretación de los datos, la pretensión fundamental es intentar caracterizar los constructos derivados del análisis factorial a partir de una teoría de los procesos cognitivos frente a la práctica de desarrollar una teoría de los procesos cognitivos a partir de los resultados en análisis factoriales. Una de las características fundamentales, como apunta Martínez Arias (1982), es la de considerar los factores como "variables dependientes» que deben explicarse por medio de aspectos o características más elementales del procesamiento de la información, y no como "variables independientes" tal y como han sido consideradas con anterioridad.

Evidentemente, una estrategia de análisis racional, sin elaboraciones empíricas, resulta altamente subjetiva y difícil de diseñar. No obstante, a modo de ejemplo podemos sintetizar de alguna manera, el tipo de estudio llevado a cabo por J. B. Carroll (1976, 1983), a través de los siguientes pasos:

1. Tomar como marco de referencia un modelo de procesos cognitivos. Su finalidad es la interpretación y caracterización de constructos diferenciales hallados a través del análisis factorial, de acuerdo con el modelo elegido. Carroll (1976), por ejemplo, eligió el modelo de Memoria Distribuida de Hunt (1971) al que le añadió el concepto de "sistema de producción» de Newell y Simon (1972).

2. Elección de los factores, objeto de análisis. Carroll seleccionó los 24 factores medidos a través de 74 tests, que estaban recogidos en el Kit de Tests de Referencia para Factores Cognitivos de French, Ekstrom y Price (1963). Posteriormente (Carroll, 1983) realizó un estudio comparativo entre diversos listados de factores - Cattell (1957), French (1951), French, Ekstrom y Price 91963) y Guilford (1963)-.

3. Desarrollar un sistema uniforme para clasificar las características de las tareas presentadas por los items de cada tests. A tal fin, Carroll asumió que las tareas cognitivas usadas en los estudios de análisis factorial eran necesariamente complejas, desde el punto de vista del procesamiento de la información, y que los factores simplemente tendían a caracterizar o resaltar algunos aspectos de dicho procesamiento en el que existían notables diferencias individuales. Con este principio evitaba la asunción de que los fac- 
tores podían ser clasificados mediante n-sistemas taxonómicos. El esquema de clasificación incluía:

a) Tipo de estímulo presentado.

b) Tipo de respuesta abierta requerida.

c) Estructura de la tarea.

d) Operaciones y estrategias requeridas.

e) Aspectos temporales de la operación o estrategia.

f) Tipo de almacén de memoria implicado.

4. Análisis de los factores, en función del modelo elegido y del mencionado esquema de clasificación, a fín de específicar las fuentes potenciales de las diferencias individuales en dichos factores.

Pese a ser ésta una forma de trabajo altamente subjetiva y especulativa, contiene un gran valor heurístico como generador de hipótesis sobre las diferencias de procesos psicológicamente significativos entre individuos.

En concreto, Carroll (1981) ha identificado una lista tentativa de diez tipos de componentes cognitivos: CONTROL, ATENCION, APREHENSION, INTEGRACION PERCEPTIVA, CODIFICACION, COMPARACION, FORMACION DE LA COREPRESENTACION, TRANSFORMACION y EJECUCION DE LA RESPUESTA.

Carroll es consciente de que estos componentes no tiene por que ser mutuamente exclusivos, pero afirma que son lo suficientemente diferentes como para constituirse en el fundamento de un análisis del procesamiento de la información de la ejecución de las tareas inteligentes.

\section{Estrategia de Simulación por Ordenador}

Antes de comentar esta estrategia, debemos hacer algunas consideraciones sobre la Simulación y la Inteligencia Artificial. Tal y como expone Vega (1984) «la simulación y la Inteligencia Artificial son dos tipos de programas de ordenador capaces de mimetizar dominios concretos del comportamiento inteligente humano" (p. 52). Aunque no puede hablarse de ambas como métodos empíricos específicos, sí nos interesa tratarlos en la medida en que pueden ser tomados como métodos de depuración formal de teorías, y por tanto, como estrategias de investigación.

Por otra parte, aunque ambas son programas de ordenador, existen profundas diferencias entre ellas. La Inteligencia Artificial (IA) enfatiza el producto, es decir, pretende lograr el procedimiento más parsimonioso y eficaz para lograr un objetivo máximamente eficaz. La Simulación enfatiza el proceso, y pretende representar la generación de un comportamiento por medio de procesos que son equivalentes, a cierto nivel (¿funcional y/o computacional?), a los del individuo humano. Es decir, pretende que el programa «se parezca» al sistema cognitivo humano (Vega, 1984).

Jerry Fodor (1968) se plantea qué tendría que hacer la simulación mediante una máquina, para que dicha simulación pudiera servir de explicación de cómo son capaces de hablar las personas con una determinada intención. Este autor afirma que «para que una simulación adecuada constituya una explicación adecuada, deben darse estas dos cosas: que las conductas accesibles a la máquina se correspondan con las accesibles al organismo, y que los procesos por los que la máquina produce su conducta si- 
mulen los procesos que utiliza el organismo para ello" (p. 175). El fundamento que sustenta la creencia de que el repertorio conductual de la máquina se corresponde con el del organismo es el logro de una correspondencia entre los subconjuntos de esos repertorios observables directamente. Pero es necesario tener en cuenta que los datos obtenidos por inducción no entrañan las hipótesis que apoyan. De esto se sigue que ninguna simulación satisfactoria puede servir de criterio de suficiencia para especificar el grado de explicación de un determinado modelo psicológico.

Escépticamente, John Searle (1985) se basa en el hecho de que las operaciones del ordenador se definen sintácticamente, por definición, para negar la tesis de su capacidad para duplicar los rasgos mentales de los individuos - p.e. pensamientos, sentimientos, emociones, et.-. En opinión de este autor, la distinción clave debe establecerse entre simulación y duplicación. Para él, «ninguna simulación constituye, por sí misma, duplicación» (p. 43).

Dejando a un lado estos argumentos, un tanto pesimistas respecto a la simulación por ordenador de los procesos cognitivos, que por otra parte, son una buena introducción a la presente temática, cabe hacerse la siguiente pregunta: ¿Cuál sería el papel de las estrategias de simulación en el estudio de las diferencias individuales? Kjeldergaard (1967) propone que, por lo que se refiere a las diferencias individuales, las estrategias de simulación y de IA deberían contemplarse como extremos de un continuo, tal y como se muestra en la Figura 1:

\section{FIGURA 1}

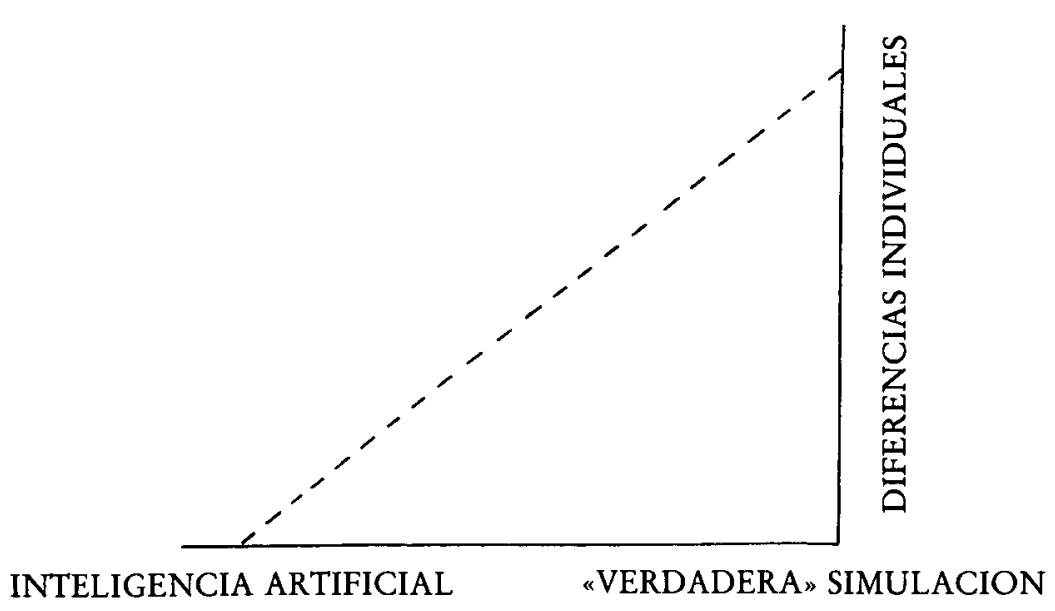

Relación entre el grado de implicación de las diferencias individuales en el continuo inteligencia artificial-simulación

En un extremo se encontraría la IA que, al considerar como objetivo la solución opcional más parsimoniosa y eficaz de un problema, imposibilitaría prácticamente consideraciones directas de las diferencias individuales. En el otro extremo, se hallaría la hipotética verdadera simulación, es decir, la que mimetizaría el comportamiento cognitivo humano; siendo éste variable inter e intra individuos necesitaría, no solo tomar en cuanta las diferencias, sino también incorporarlas dentro de la simulación. 
A pesar de este panorama, la IA puede servir como heurístico para el estudio de las diferencias individuales. Como apuntan Dhen y Schank (1982), los primeros estudios de IA se interesaron por la cuestión de las diferencias humanas en el sentido de que importaba conocer los prerequisitos necesarios para ejecutar tareas que, únicamente las personas muy hábiles podían ejecutar. No obstante, estos aspectos fueron muy prematuros al no contar con una teoría subyacente de la Inteligencia Normal sobre la que basar los resultados obtenidos. A pesar de esto, la IA sirve como fuente de sugerencias en tres sentidos:

1. La evolución histórica de los programas de IA desde los «menos inteligentes» hasta los más desarrollados, aunque no reflejan perfectamente los grados de inteligencia humana, aportan una serie de elementos que ocurren o no ocurren en varios grados, o tipos, de conducta inteligente.

2. Los modelos de IA son lo suficientemente explícitos en procesos y fuentes de conocimientos, como para ser dúctiles al análisis y determinación de lugares donde podría ocurrir la variación (p.e. fuentes de diferenciación).

3. Los programas de IA pueden ser utilziados como aparatos con los que poner en juego variables de inteligencia hipotetizadas, y determinar las diferencias que producen.

Por lo que respecta a la simulación, la panorámica es compleja y los trabajos con programas escasos. Como ya hemos comentado anteriormente, la simulación pretende representar la generación de un comportamiento mediante procesos «equivalentes» a los de un sujeto humano. Es decir, la simulación pretende elaborar un programa que sea una buena teoría psicológica (Vega, 1984). No obstante, este panorama - escrito en un lenguaje formal de ordenador, BASIC, ALGOL, etc., que no se asemeja a la teoría- viene a ser un test, no de una teoría general, sino de un dominio específico, como las imágenes mentales (Kosslyn, 1984), o una tarea determinada, ya sea ésta sencilla como la de «verificación de sentencias» -ideada por Clark y Chase (1972)_ o compleja como el «completamiento de Series de Letras" (Kotovsky y Simon, 1973). Su ventaja es que, al operar "paso a paso" sobre un diseño de ejecución de, por ejemplo, una tarea, obliga a ser muy explícitos para lograr las predicciones establecidas y, por tanto, nos permite aislar aquellos componentes que, por ser flexibles, están abiertos a un rango de variación entre individuos (Kosslyn, 1980, Kosslyn et al., 1984).

Parece lógico suponer que, tanto las propiedades estructurales de un almacenamiento de información, como las estrategias adquiridas previamente para percibir, identificar, etc., dicha información, sean diferentes y puedan ser representadas de forma diferente. Por otro lado, también parece lógico suponer que las reglas funcionales que describen las transformaciones y las manipulaciones de los datos almacenados, puedan ser organizadas diferencialmente por diferentes sujetos. Todo ello en su conjunto conforma un sistema altamente complejo donde, si bien el isomorfismo físico «hombre-máquina) sea irrelavante, la ejecución del programa debe reproducir los parámetros básicos del comportamiento humano - tiempo de reacción, probabilidad de respuesta, etc.- en la ejecución de una tarea.

Como el resto de las estrategias, el uso de la simulación para el estudio 
de las diferencias en cognición, pretende establecer una teoría que guíe los datos empíricos, evaluándola a través de un programa de simulación. Esta estrategia de simulación, desde el punto de vista de la Psicología Diferencial Cognoscitiva, puede ser de dos tipos: estrategia de simulación normativa, y estrategia de simulación criterial. En cualquiera de los casos, el programa de simulación, al proceder paso a paso, permite aislar componentes del sistema cognitivo que pueden ejercer una función diferenciadora entre los individuos en una tarea dada y a la vez, evaluar la teoría propuesta sobre dicha tarea. La mecánica general de la simulación en sí es sencilla: consiste en elaborar un programa de ordenador que dé cuenta de los pasos necesarios para la ejecución de una tarea, en función de una teoría propuesta sobre cómo la ejecutarian sujetos humanos, y contrastar los resultados de la simulación con protocolos obtenidos de dichos sujetos, observando la bondad de ajuste entre ambos datos.

La simulación normativa parte de un marco teórico donde las diferencias humanas adquieren su sentido desde las desviaciones de los sujetos respecto de alguna medida de tendencia central. Se pretende simular el comportamiento de un grupo, y por tanto el programa se diseña en base a datos normativos y un esquema de clasificación de respuesta, a partir de los cuales, se pueden tabular y poner a prueba los aciertos y los errores, con estadísticos de frecuencias respecto de las predicciones que se establecen en el modelo.

En términos generales, la forma de proceder sería como sigue:

1. Establecer el objetivo de simulación; por ejemplo Kjerdergaard (1967), en su experimento con el programa APE - Association Producing Engine-, pretendía representar el comportamiento diferencial en la asociación libre de palabras.

2. Hipótesis sobre el comportamiento diferencial en el dominio o tarea elegida.

3. Datos empíricos de sujetoshumanos reales sobre dicho dominio o tarea.

4. Elaboración de una teoría psicológica sobre la forma de proceder de los sujetos respecto de la tarea, y los componentes responsables de las variaciones en los productos.

5. Diseño del programa basado en datos normativos.

6. Ejecución del programa.

7. Observación del ajuste en función del contraste entre los datos empíricos y los resultados de la ejecución del programa.

8. Toma de las decisiones pertinentes en cuanto a posibles nuevas hipótesis o reformulaciones de las teorías o del diseño del programa.

Gregg (1967) critica este tipo de estrategia sobre la base de que: a) no es una auténtica simulación, ya que ésta pretende representar el comportamiento de un grupo, y la auténtica simulación es «un" modelo para «un» solo individuo; y b) que estos modelos de simulación basados en datos normativos son, en sì mismos, más una teoría acerca de cómo deben comportarse los datos, que una teoría sobre el comportamiento diferencial de los sujetos.

Los estudios sobre simulación normativa fueron abandonados en nuestra opinión prematuramente, en favor de la simulación criterial. No obs- 
tante, la simulación normativa es útil porque pretende explicar el comportamiento de un grupo de individuos, por lo que gana en amplitud explicativa, frente a la simulación criterial, que restringe su rango de descripción y explicación a un solo individuo, es decir, disminuye su potencial generalizador.

Las estrategias de simulación criterial, por su parte, asumen una teoría de carácter general sobre el comportamiento humano, a partir de la cual pueden observarse las variaciones de los sujetos en las ejecuciones de una tarea específica. Por tanto, el estudio de las diferencias sería una prolongación del programa de simulación que representa un modelo para un solo individuo, el cual se toma como criterio. La importancia del estudio sobre las diferencias se manifiesta en problemas a cuya solución puede llegarse por diferentes vías, $y$, ante los cuales, diferentes sujetos pueden usar procesos diferentes para resolverlos. Como apunta Simon (1976): «si sólo conocemos que un sujeto puede resolver el problema de la Torre de Hanoi con tres discos, no podemos decir cuál de estas estrategias -método de repetición, método recursivo, método perceptual y método de patrones-, (o alguna otra), está usando».

Para elaborar una estrategia de simulación de este tipo, se pueden seguir las siguientes fases: una primera, en la que se pondría a prueba el modelo general de simulación, y la segunda, en la que se realizaría un acercamiento al problema de las diferencias. La primera de estas seguiría en líneas generales los pasos descritos por Vega (1984, p. 67):

1. Hipótesis psicológica.

2. Datos empíricos con sujetos humanos.

3. Teoría psicológica.

4. Diseño del programa de simulación.

5. Ejecución del programa.

6. Planteamiento de nuevas hipótesis y predicciones.

La segunda fase comenzaría cuando la simulación se comporte según las predicciones de la teoría, alcanzando un nivel de suficiencia de ajuste. A partir de este punto, y contrastando el modelo con los protocolos de los individuos reales, se hipotetizan las fuentes de variación de aquellos individuos que no han resuelto la tarea, o lo han hecho con fallos, y de aquellos que, aún resolviendo la tarea adecuadamente, se sospecha que han utilizado estrategias diferentes para ello. Las formas de proceder son diversas y oscilan entre la mera constatación de aquellos que muestran tales diferencias, hasta la creación de modelos que simulen la ejecución de la tarea con estrategias alternativas. El problema fundamental de este tipo de estrategia de simulación es su falta de generalidad, al corresponder al modelo de una tarea representando un individuo.

En resumen, ambas estrategias pretenden dar un fundamento teóricocientífico a los datos mediante contrastación con un programa de simulación. Las diferencias estriban en dos puntos fundamentales. Con la simulación normativa se pretende representar la ejecución de un grupo, basándose en datos normativos; mientras que con la simulación criterial se pretende representar la ejecución de un solo sujeto, y toma a ésta como criterio para descubrir las diferencias. Por otro lado, la primera de ellas plantea la duda de si es una teoría sobre el comportamiento interindividual en la 
ejecución de la tarea, o si es una teoría acerca de cómo deberían comportarse los datos; y la segunda es una teoría sobre la ejecución de un «sujeto-esquema-general». De cualquier forma, ambos tipos de estrategias son recursos de investigación altamente valiosos para el análisis de tareas, y este. valor se constata con mayor fuerza cuando las tareas analizadas son iguales o semejantes a los problemas incluidos en los tests psicométricos diseñados para medir constructos diferenciales, y ante los cuales los sujetos muestran sus diferencias.

\section{Estrategia de Simulación Matemática}

Los modelos matemáticos utilizados en las estrategias de simulación matemática, pretenden simular la realización de una tarea generando una serie de ecuaciones que, sustituyendo las variables pertinentes, realice predicciones ajustadas a la actuación humana en un determinado conjunto de tareas (Sternberg, 1985).

Por ejemplo, en el análisis de latencias de respuestas de una tarea de resolución de problemas, el principio que subyace es que los procesos cognitivos operan en tiempo real, y que tales tiempos son aditivos. El TR total, por tanto, se podría descomponer para averiguar la duración y organización de los procesos bajo análisis. El procedimiento general sería:

a) Analizar la tarea. Se suele utilizar el método thinking aloud (pensamiento en voz alta).

b) Construir condiciones experimentales que requieran procesos ligeramente diferentes o diferentes frecuencias de determinado proceso, de acuerdo a lo especificado en el análisis de la tarea.

c) Formular ecuaciones para expresar la latencia de cada condición en función de los procesos implicados.

d) Medir las latencias de respuesta en cada condición.

e) Comparar las latencias en las distintas condiciones para determinar la duración de cada proceso.

Mumaw, Pellegrino y Glaser (1980) realizaron una investigación con sujetos adultos, utilizando una tarea de resolución de problemas extraídos del MINNESOTA PAPER FORM BOARD TEST -que proporciona una medida de la aptitud espacial- . Se les pedía a los sujetos que determinasen cuál de los 5 cuadrados podía construirse con las piezas que se presenta en el extremo inferior de la Figura 2.

Los procesos hipotetizados por los autores, por lo que se refiere a la secuencia de resolución de la tarea, fueron los siguientes:

En primer lugar, los sujetos CODIFICAN una de las piezas.

En segundo lugar, BUSCAN una pieza que se pueda emparejar.

En tercer lugar, las piezas se ROTAN mentalmente hasta lograr que se ajuste a la pieza correspondiente.

Por último, se COMPARAN para determinar si encajan.

Si no encajan, el sujeto concluirá que no son útiles para formar el cuadrado.

Si encajan, se repite la secuencia CODIFICAR-BUSCAR-ROTARCOMPARAR hasta que se hallan puesto a prueba todas las piezas, y se responda. 
Figura 2

I.

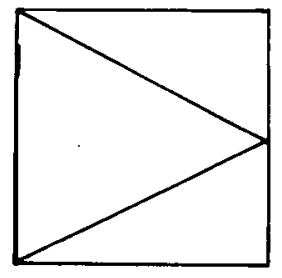

2.

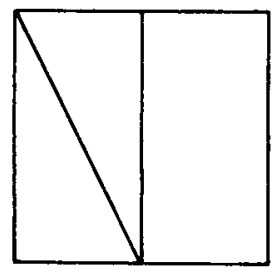

3.

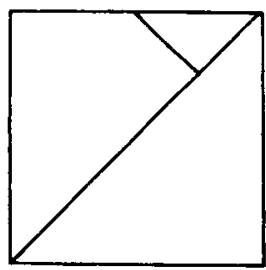

4.

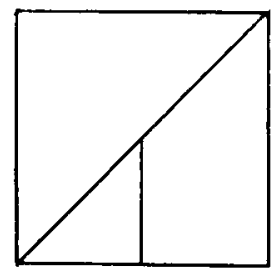

5.

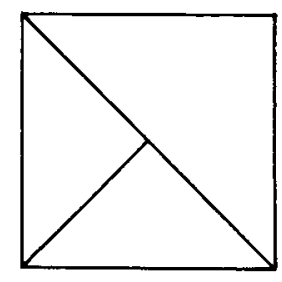

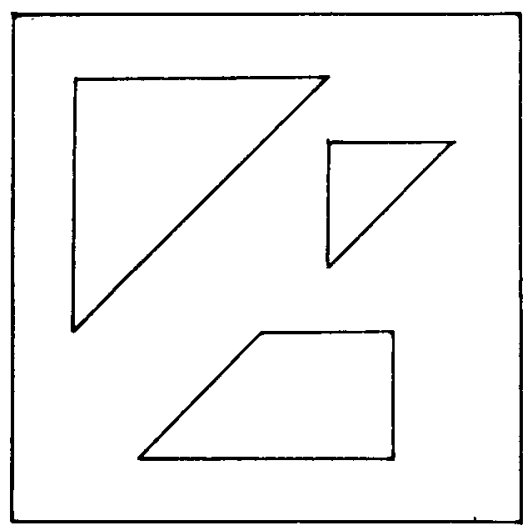

Minnesota paper form board test, para medir la capacidad espacial 


\section{4}

Por simplicidad, sólo consideraremos las predicciones respecto a las piezas que puedan reunirse para formar el cuadrado. De acuerdo con el modelo propuesto por Mumaw, Pellegrino y Glaser (op. cit.), el TR (Tiempo de Reacción) para el problema quedaría definido, o vendría determinado, de la siguiente manera:

$$
\mathrm{TR}=\mathrm{N}(\mathrm{c}+\mathrm{b}+\mathrm{r}+\mathrm{Cp})+\mathrm{k}
$$

siendo $\mathrm{N}=$ número de piezas; $\mathrm{c}=$ tiempo de codificación; $\mathrm{b}=$ tiempo de búsqueda; $r=$ tiempo de rotación; $\mathrm{C} p=$ tiempo de comparación; $\mathrm{k}=$ constante que representa los procesos supuestamente iguales para todos los problemas, como p.e. el tiempo requerido para dar una respuesta observable, una vez conluida la operación mental $c+b+r+c p$.

Para valorar el modelo, los autores crearon diferentes problemas variando las dimensiones relevantes del mismo (p.e. variando el número de piezas de 2 a 6; de acuerdo al modelo, el TR aumentará linealmente con el número de piezas). Como resultado de esta variación en las dimensiones se obtuvieron 300 problemas. Los resultados obtenidos fueron los siguientes: a) análisis grupal: correlación $+.965 ; b)$ análisis individual: correlación mayor o igual a .90 (correlación empírica predicha).

Los procedimientos para identificar la varianza sistemática de la que nos da cuenta el modelo es útil a la hora de decidir si cualquier modelo puede dar cuenta de una gran proporción de varianza para un conjunto determinado de datos. Este punto lleva a dificultades de interpretación de la correlación, en cuanto a su relevancia psicológica. Cuando la cantidad de información que debe ser procesada varía considerablemente en los distintos problemas, o cuando se evalúa un único modelo, los investigadores deben considerar la posibilidad de que los valores altos de correlación sean espúreos. Pueden encontrarse una serie de dificultades a la hora de comparar modelos contrapuestos. Si dos modelos se relacionan jerárquicamente, los resultados pueden ser comparadados estadísticamente con el fin de determinar si los parámetros adicionales del modelo más complejo contribuyen significativamente a la ecuación de regresión. Cuando no hay relación entre los modelos, no existe base para realizar comparaciones puramente estadísticas.

En algunas ocasiones, los modelos se evalúan analizando la significación de un grupo: no obstante el mejor procedimiento es evaluar modelos para individuos. En resumen, en ocasiones, y no precisamente en las menos, se da escasa interpretación psicológica a los componentes de un modelo.

\section{Estrategias de comparación de grupos}

La estrategia de comparación de grupos, ya sean utilizados éstos para conocer las diferencias existentes entre ellos, o como estrategia de investigación para descubrir componentes diferenciadores de la cognición humana, es una de las más complejas e intrincadas de la investigación en cognición. Esta complejidad ha venido siendo puesta de relieve por diversos autores desde campos tan diversos como los estudios transculturales (Coles y Means, 1981), el déficit cognitivo en esquizofrénia (Chapman y Chap- 
man, 1973), el déficit diferencial en sujetos retrasados (Chapman y Chapman, 1985), o más genéricamente en el estudio de las diferencias en procesos cognitivos (Baron y Treiman, 1980). Pese a la mencionada complejidad y las múltiples dificultades metodológicas que estos conllevan, el uso de tal estrategia se ha venido haciendo cada vez más creciente y en el momento actual es de gran valor tanto en el terreno de las diferencias individuales en cognición como en el terreno del refinamiento de hipótesis generales.

Tradicionalmente, la perspectiva psicométrica de la Psicología Diferencial se interesó en la estrategia de comparación de grupos en función de variables como el sexo, raza, cultura, clase social, grupos profesionales, etc., que se hipotetizaban como factores explicativos cuasi-causales, en potencia o subyacentes, de las diferencias en aptitudes cognitivas o de personalidad. Como apuntan Cole y Means (1981), si bien estos estudios han sufrido un fuerte descrédito como forma de demostrar causación, han desempeñado un papel importante al estimular el interés en el estudio comparativo de la cognición. En efecto, el valor de estos estudios ha estado, no tanto en su valor explicativo como en el descriptivo. Daban respuesta a la pregunta de «cuanto» diferían los grupos humanos clasificados con criterios morales tales como sexo, raza, cultura, nivel intelectual, etc., respecto a sus puntuaciones en tests psicométricos o medidas morales de la eficacia o el estilo cognitivo. No obstante, la pregunta sobre el "cómo" diferian los sujetos en sus ejecuciones no podía ser respondida y ni siquiera se podía pretender ofrecer una vía de respuesta desde esta perspectiva. La ausencia de un marco teórico que fuera guía de resultados así como los problemas derivados de la imposibilidad de asignar los sujetos al azar a las condiciones en un experimento, o la confusión entre los criterios de selección de los grupos y las variables independientes no manipulables, entre otros, han sido y siguen siendo las piedras de toque de los estudios comparativos de grupos desde el marco psicométrico de la Psicología Diferencial.

Desde la perspectiva de la psicología diferencial cognoscitiva, los estudios de comparación de grupos nos permitirían, al menos teóricamente, establecer las fuentes de diferenciación de las variaciones en los productos ante una tarea, o establecer los diversos modos de operar de los sujetos por los que se puede acceder a un producto, bien diferencial, o con una variación mínima, a un nivel de análisis dado. Las tareas sobre las que se realizan los trabajos requieren un análisis, como mínimo implícito y como más deseable, explícito, ya sea mediante la adscripción a un modelo lógico-racional teoricamente derivado de procesamiento de la información, o como resultado de un modelo matemático o de simulación. Los criterios de selección de grupos necesitan ser más precisos, y el investigador debe proceder a la manipulación de estrategias o contenidos cognitivos.

Por todo esto, la investigación diferencial cognoscitiva no se ocupa de la caracterización de grupos en sí, aunque sea muy legítimo e incluso deseable realizar trabajos de este tipo; sino que los grupos son utilizados como estrategia de trabajo para el estudio diferencial de la cognición. Una de las razones es que existen características derivadas de la dificultad de trabajar sobre los problemas de la disponibilidad, accesibilidad, o déficit general de algunos componentes cognitivos. Esto hace necesario la utilización de sujetos que presenten una carencia a priori en estos componentes. La ausencia de un canal sensorial, y su influencia en la modalidad representacional 
(p.e. déficit visual en ciegos de nacimiento) sólo se puede estudiar mediante una estrategia de comparación de grupos. Otros aspectos son, por ejemplo, la influencia de la velocidad de procesamiento en otras variables cognitivas, o simplemente en la ejecución de tareas complejas, pertenece al mismo grupo de problemas por los que se tiene que elegir este tipo de estudios de comparación de grupos.

Los grupos que se suelen comparar son: 1) sujetos con déficits sensoriales/sujetos normales (ciegos, sordos, etc.), 2) sujetos con retraso mental/sujetos normales, para el estudio de la accesibilidad y disponibilidad de procesos y/o estategias, 3) grupos de distintas culturas para comprobar la generalizabilidad de patrones de estrategias de resolución de problemas, por ejemplo, 4) sujetos superdotados/sujetos normales para el estudio de componentes de máxima eficacia, 5) expertos/novatos para el estudio de la influencia de la base diferencial de conocimientos, 6) expertos/expertos de distintos campos de conocimiento para analizar los componentes diferenciales de ejecución de alto nivel, y un largo etcétera que podría incluir amnésicos/normales, u otras características, así como múltiples combinaciones de estos grupos.

Uno de los problemas a los que nos enfrentamos en este tipo de trabajos, común a toda investigación en cognición, hace referencia a la definición de componente cognitivo o parámetro de procesamiento. Desde la metodología experimental, un componente cognitivo o parámetro de procesamiento no puede ser observado directamente, sino sólo inferido a través de las variaciones de respuesta entre dos condiciones. Por tanto, podríamos decir, en estricta interpretación, que dichas diferencias entre condiciones es un indicador de la "validez aparente» del componente o parámetro en cuestión (Baron y Treiman, 1980). No obstante, en cognición un parámetro o componente cognitivo no sólo es definido en términos de dichas diferencias, sino también referido a un modelo teórico, por lo que su validez es referida a un modelo evaluado previamente. Esto significa conocer el tipo de función -lineal, monotónica, etc.- entre el componenete teórico hipotetizado y los datos, en términos de probabilidad de respuesta o tiempo de reacción, de la variable dependiente (cf. Loftus, 1978). Por tanto, cualquier estudio diferencial que asuma tales componentes de diferenciación, o está referido a un modelo teórico, implícito o explícito, o la validez del componente hipotetizado como responsable de la variación, sólo sería aparente.

Las estrategias de comparación de grupos abarcan un número y variedad de investigaciones muy considerables y, por tanto se hace difícil su sistematización y concepción de un diseño guía general. Los objetivos de estas investigaciones son diversos y los procedimientos se combinan. No obstante, intentaremos ofrecer un esquema de procedimiento general en el cual nos basaremos para realizar ulteriores especificaciones.

A. Adopción o diseño de un micromodelo de cognición relevante para el aspecto que se pretende analizar. Este modelo nos servirá de guía para establecer de forma clara y precisa el fenómeno objeto de nuestro estudio, evaluar las posibilidades explicativas de la teoría como fuente provisional de explicación de las diferencias hipotetizadas, bien mediante la realización de los análisis pertinentes, o el análisis y selección de investigaciones pre- 
vias, y. por último nos permite conocer en qué medida la tarea que pretende representar el fenómeno es susceptible de análisis explícito (Rohwer, 1976).

B. Elaboración de la hipótesis diferencial: estos estudios parten de la necesidad de descubrir el llamado déficit diferencial. Sin embargo, en Psicología Diferencial Cognoscitiva, el objetivo es conocer la relevancia que tiene uno o más componentes del sistema cognitivo para la ejecución general. En estos términos, las hipótesis van encaminadas a la confirmación de la responsabilidad relativa de un componente cognitivo específico. Dado que uno de los problemas es el acceso directo a dichos componentes, parece conveniente distinguir entre lo que son hipótesis explicativas, de carácter teórico,, e hipótesis instrumentales, derivadas de la tarea o acciones que se pretenden realizar, de tal manera que preservemos la posibilidad de confundir un fallo en la investigación atribuible a un inadecuado análisis de la tarea, o bien a una modificación de la misma, con el fallo atribuible al componente cognitivo hipotetizado como fuente de diferenciación. Por otra parte, la formulación de estas dos hipótesis nos permiten establecer el tipo de relación entre componente hipotetizado y los datos derivados de las manipulaciones (Loftus, 1978).

C. Selección de Tareas: consiste en elegir tareas, por lo regular de laboratorio, que tras investigaciones han demostrado que reflejan la presencia de un determinado componente cognitivo. Por ejemplo, la tarea de Saul Sternberg (1969) refleja un componente cognitivo de manipulación de la información en la memoria activa. La selección de tareas, sin embargo, es uno de los capítulos de este tipo de investigaciones, ya que condicionan tanto los tipos de estrategias a elegir como el margen de interpretación que podemos obtener en los resultados. La selección de tareas puede realizarse en función de si obedecen a un modelo explícito o implícito de la misma. Por otra parte, también la tarea puede elegirse en función deltipo de estrategia mnemónica requerida o el tipo de conocimiento implicado en la misma (Brown, 1975).

D. Selección de Grupos: En cuanto a la elección de un criterio de selección de grupos, Rohwer (1976) plantea que nos podemos encontrar con dos tipos de criterios generales, y diversos tipos de indicadores:

1. Criterio simple: consiste en identificar al menos dos poblaciones, una que ejecute adecuadamente la tarea objeto de análisis, y otra que la ejecute con dificultad.

2. Criterio complejo: implica modelos de regresión de diseños y análisis. El paso supone el uso de un indicador que asigne valores a las personas en una escala continua de predicción sobre la subsecuente ejecución de una tarea dada.

Los tipos de indicadores de características de los sujetos son varios y, en términos generales, pueden dividirse en indicadores intrínsecos o extrínsecos, dependiendo de la medida en la que la característica de la que es indicador esté directamente implicada, o no, en la ejecución de nuestro interés. Los indicadores extrínsecos quizá sean los más conocidos por ser los normalmente utilizados en la Psicología Diferencial Psicométrica; entre ellos se encuentran la edad, el sexo, el status socioeconómico, las puntuaciones en tests, etc. Los indicadores de características intrínsecas son más 
difíciles de establecer siendo, no obstante, los más interesantes para las estrategias de comparación de grupos. La dificultad estriba, ante todo, en que dichos indicadores pretenden ser medidas increíbles de diferencias en componentes específicos, los cuales se toman como responsables de las diferencias en los sujetos. Un aspecto a tomar en cuenta en los indicadores intrínsecos es su sensibilidad a la variación. Dicha sensibilidad a la variación debe obedecer a una sola dirección, es decir, ser sensible sólo a las variaciones en los componentes hipotetizados y no a otros. Los indicadores intrínsecos a su vez pueden dividirse en indicadores puros y globales. Por ejemplo, un indicador puro puede ser la tendencia al procesamiento semántico de palabras, frente al indicador global de ejecución en una lista de nombres comunes cuando la tarea criterio es el aprendizaje de la información de la identidad de dichos nombres. Por lo regular podemos decir que los indiciadores puros son preferibles a los globales o burdos, de la misma manera que los intrínsecos a los extrínsecos.

E. Comprobación de hipótesis: una vez aplicadas las tareas se plantea la dificultad de la verificación de la hipótesis, ya que es difícil llegar a este punto, puesto que los sujetos no están asignados al azar a las condiciones. Ante este problema de difícil solución, los investigadores establecen trabajos convergentes para dar más solidez a los resultados, aplican métodos iterativos de análisis, o bien optan por estudios de simulación de diferencias que si bien pueden considerarse experimentales, con todas las ventajas que ello conlleva de asignación de los sujetos al azar a las condiciones y manipulación de las variables independientes, existe siempre el problema derivado de la naturaleza del objeto de estudio, de saber si al inhibir un proceso o estrategia para simular estas diferencias no estaremos afectando a otros procesos o estrategias de forma no controlada.

A continuación, pasaremos a comentar las distintas subestrategias de comparación de grupos. El criterio adoptado para su diferenciación es el mismo que plantean Cole y Means (1981) en función del modelo de análisis de tarea, a saber: aquellas que obedecen a modelos implícitos (quizá no adecuadamente definidos), y aquellas que obedecen a modelos formales directamente relacionados con las tareas. Los modelos implícitos, por su parte, implican variaciones instituidas en las tareas cognitivas que pretenden representar el proceso y/o la evaluación del impacto que tienen dichas variaciones en los distintos grupos de los sujetos. Sin embargo, si bien estas tareas nos proporcionan información sobre los sujetos de rendimiento normal, la información que recibimos de aquellos sujetos con rendimiento deficitario es que, o bien no ejecutan a cierto nivel, o bien que no tienen cierto atributo necesario para dicha tarea, pero escasamente nos informan de qué es lo que pueden hacer estos últimos sujetos. En los modelos explícitos, el investigador pretende desarrollar algunos modelos de ejecución cognitiva cuyo objetivo es representar, bien diferentes estrategias, bien diferentes niveles de conocimiento, e intentar dirigir la tarea de tal manera que la conducta de un sujeto revele qué modelo, si es que este existe, corresponde a sus procesos o a sus mecanismos en la realización de la tarea. Estos modelos pueden manifestarse en forma de análisis lógico-racional, simulación matemátiva, o simulación por ordenador. En cualquier caso, ambos tipos de estrategia depende de la descomposición al original 50. 
Subestrategias de comparación de grupos con tareas de modelo implícito

Subestrategia de comparación de patrones de ejecución:

Su objetivo es hacer frente al probleima de encontrar procesos diferenciales entre grupos, a partir de tareas que carecen de una teoría que relacione la ejecución con los procesos subyacentes. Si bien lo ideal en estos casos sería contar con dichas tareas, esperar a ello no siempre en un buen método, ya que supone creer en la existencia del "análisis correcto" de dicha/s tarea/s, y que este análisis además pueda ser independiente, tanto de los sujetos como de las circunstancias que rodean a la tarea. Su característica más importante es que se centra en los patrones de ejecución, y no en el nivel de ejecución. El procedimiento para este tipo de investigaciones es el siguiente (Figura 3).

FIGURA 3

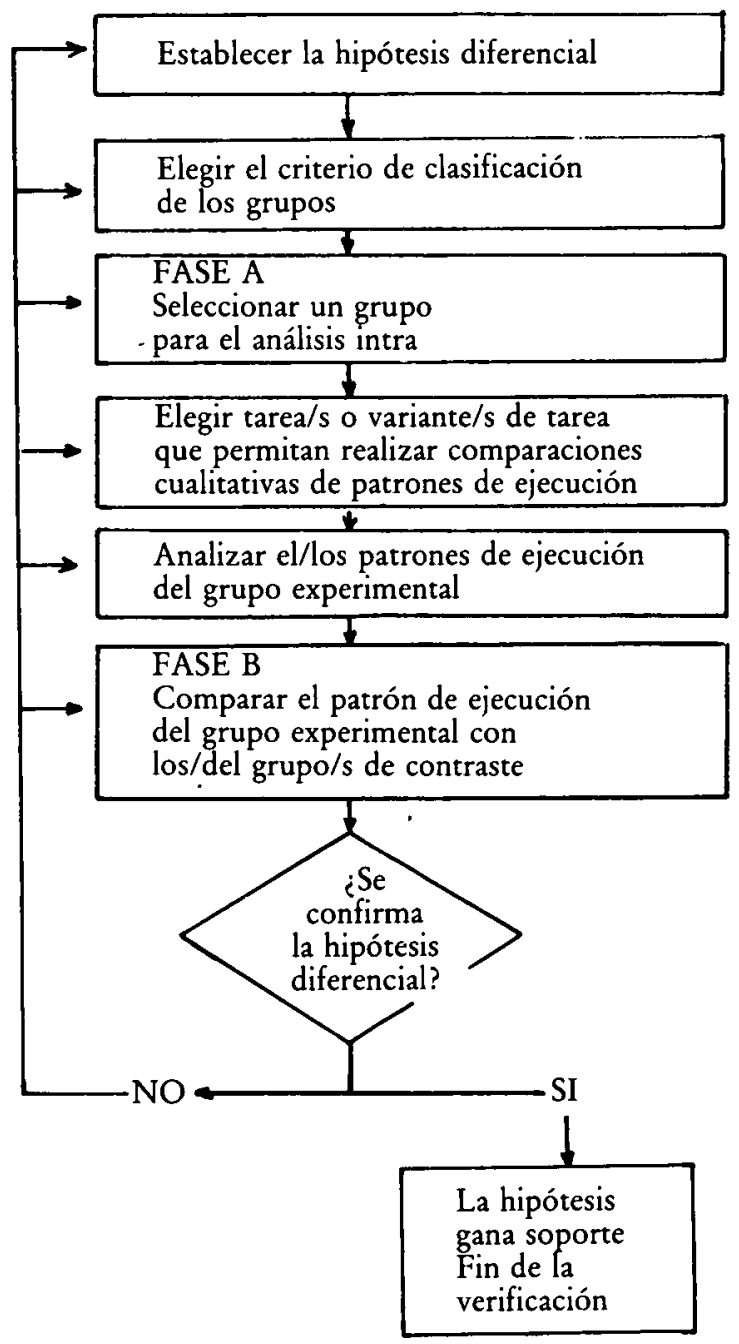

Estrategia de investigación basada en patrones de ejecución 
En primer lugar se establece la hipótesis diferencial, lo que supone especificar los componentes que subyacen a las diferencias de grupo. Evidentemente, en estos casos, dichas suposiciones se basan en investigaciones previas, o bien en observaciones realizadas por los investigadores. Posteriormente se elige el criterio de clasificación de los grupos en función de los objetivos de la investigación. La FASE A consiste en seleccionar un grupo para el análisis intra de los patrones de ejecución. En estos casos, el grupo elegido suele ser casi invariablemente, el grupo que supuestamente va a presentar un patrón de ejecución diferenciado del grupo de referencia. A continuación se elige, bien tarea o tareas, bien variantes de una tarea que relacionadas con el fenómeno objeto de estudio, puedan permitirnos con posterioridad, su utilización en la comparación del patrón de ejecución obtenido por el grupo inicial, con el patrón del grupo de referencia. Después se procede a un análisis en función de las respuestas o las tareas, o sus variantes, el patrón de ejecución del grupo inicial, de tal manera que su consecuencia pueda ser comparada. La FASE B, por su parte, consiste en la aplicación de las tareas elegidas al grupo de referencia obteniendo un patrón de ejecución que comparado con el del grupo inicial pueda permitirnos la comprobación de la hipótesis diferencial.

Esta subestrategia no se encuentra exenta de problemas; el más importante es que al centrarse en los patrones de ejecución, y no en el nivel de la misma, pueda dar como resultado «n» fuentes de diferencias que puedan conducir a resultados de escasa utilidad.

\section{Subestrategia de interacción Grupos x Tarea:}

El objetivo fundamental de esta subestrategia es aislar un componente cognitivo diferencial en la ejecución de dos grupos diferenciados con respecto a una tarea. Su característica fundamental es que tiene en cuenta no solamente los patrones de respuesta, sino la comparación directa de grupos. Su característica principal es la utilización de al menos dos tareas. La utilización de dos tareas tiene como objetivo el que al final los sujetos sólo difieran en la tarea inicial.

El procedimiento general para este tipo de investigaciones es el siguiente (Figura 4).

En principio se elabora la hipótesis diferencial. En estos casos la elaboración de dicha hipótesis parte de la aceptación de la relación entre las tareas y el proceso que se hipotetiza como responsable de las diferencias de ejecución. Este aspecto nos conduce directamente a plantear la necesidad de establecer dos tipos de hipótesis - una conceptual y otra instrumental-, aunque normalmente sólo una de ellas se haga explícita. La hipótesis conceptual establece un mecanismo cognitivo responsable de las diferencias de grupos. La segunda establece la posibilidad de que las diferencias puedan ser debidas al instrumento en sí -es decir, a la tarea- y no a la hipótesis conceptual. No tomar en cuenta estas hipótesis puede llevarnos a errores tales como no aceptar la hipótesis conceptual ya que la tarea empleada no es correcta. En segundo lugar, se elige un criterio de clasificación para los grupos, dividiendo estos grupos en grupo/s experimental/es y gru$\mathrm{po} / \mathrm{s}$ de contraste. Hemos de recordar una vez más la necesidad de acudir a los criterios comentados en el apartado de la selección de grupos. Esto 
Posible interpretación: la Tc no está directamente relacionada
con la operación responsable de las diferencias observadas con la operación responsable de las diferencias observadas en la Te. O bien no se ha logrado la equivalencia en

\section{Posible interpretación: no todos los sujetos} del Gc utilizan la operación activada por $\mathrm{T} c$.

Posible interpretación: la Tc activa alguna operación eficaz no utilizada espontáneamente por los grupos.

Posibles interpretaciones: (a) la modificación incorporada en la Tc no es suficiente para activar adecuadamence la (b) Existe alguna fuente adicional de diferencias en

2. Tc. (c) Artificio en un fallo en proporcionas

equivalencia de tratamiento en los dos grupos.
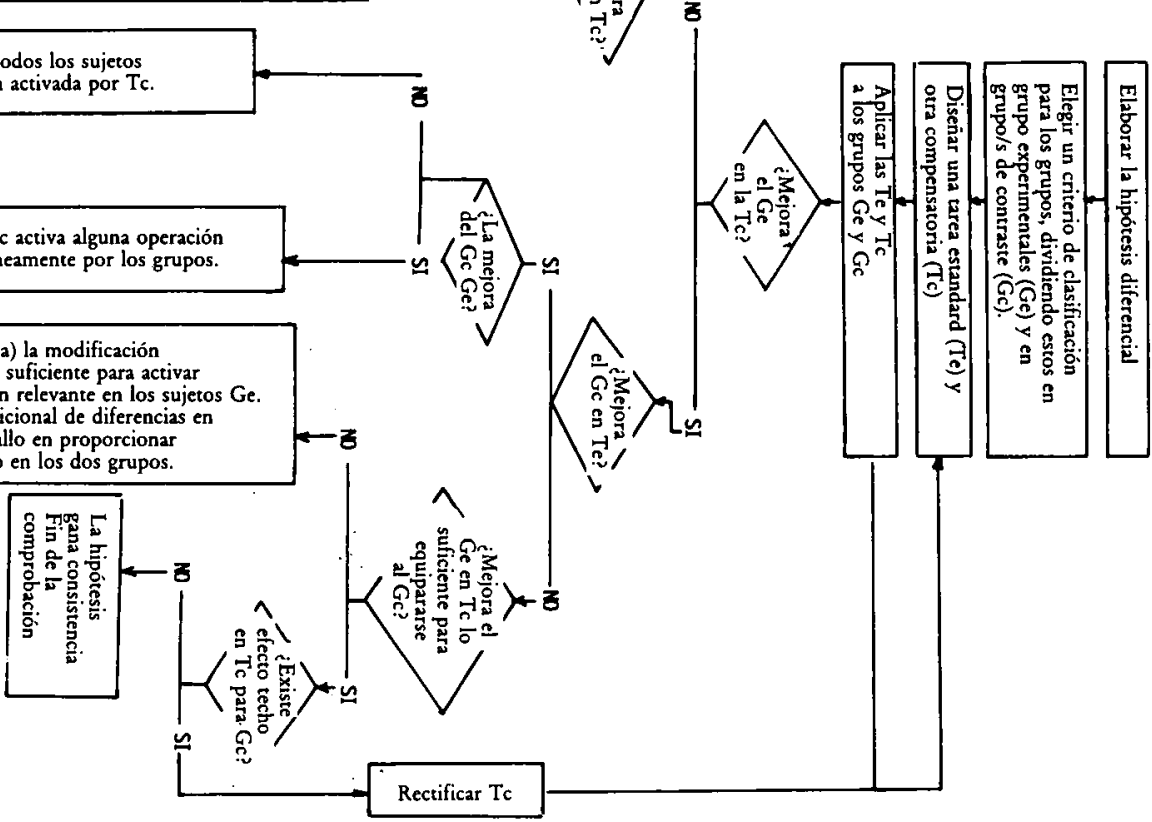
se hace especialmente importante en este tipo de investigaciones porque los grupos están compuestos por sujetos que difieren muy significativamente en nivel intelectual, nivel de pericia, etc., ya que, sobre todo en el nivel intelectual, comparando sujetos retrasados con sujetos normales, pueden presentarse déficits asociados que dificulten la correcta interpretación de los resultados. Posteriormente, se elige o diseña una tarea estandar y otra compensatoria. La importancia de esta fase es máxima, ya que la tarea compensatoria viene a ser una versión modificada de la tarea estándar, de tal manera que dicha variación inhiba o permita la ejecución de una determinada estrategia o la concurrencia o ausencia de determinados tipos de conocimiento. Estas variaciones pueden oscilar entre variaciones mínimas, o aquellas que en determinado momento puedan confundirse con entrenamientos específicos.

Rohwer (1976) distinguió entre modificaciones catalíticas o protésicas. Las primeras obedecen a ligeras modificaciones en forma de indicaciones que induzcan a los sujetos a emplear algún proceso dentro de la tarea, mientras que en las segundas dicha modificación puede llegar a ser casi una intervención directa en forma de entrenamiento. La lógica de estas variaciones es que si el nivel de ejecución superior de uno de los grupos sobre la tarea estándar proviene de algún proceso específico o estrategia, y si se modifica dicha tarea para facilitar a los sujetos su ejecución de tal manera que induzcan el proceso en los sujetos de bajo nivel, su ejecución deberá parecerse, es decir, no existir diferencias significativas, al del grupo de contraste. Una variante es diseñar modificaciones que inhiban la utilización de un proceso para simular las diferencias.

A continuación se evalúa a los grupos experimental y de contraste en las tareas estándar y compensatoria. Este es un momento delicado ya que fundamentalmente en grupos deficitarios (retrasados mentales, esquizofrénicos, amnésicos, etc.) es particularmente delicado el momento de proporcionar situaciones equivalentes para ambos grupos. Uno de los problemas puede provenir de la motivación en grupos deficitarios. Después se procede a la interpretación de resultados y a la comprobación de la/s hipótesis. La pretensión es obtener para el grupo experimental un nivel de ejecución en la tarea compesatoria que no difiera significativamente de la del grupo de contraste. Esto presenta varias alternativas: a) podemos encontrarnos con que existe una mejora del grupo experimental en la tarea compensatoria. Que además el grupo de contraste no mejore en la tarea compensatoria, cosa que debe parecer lógica si este grupo de contraste pone en funcionamiento los mecanismos cognitivos necesarios para la ejecución de la tarea estándar y la tarea compensatoria sólo es una variación que intenta facilitar la ejecución de uno de dichos procesos. Es más, podemos encontrarnos en el caso ideal de que la mejora del grupo experimental en la tarea compensatoria sea de tal calibre que permita equipararse al grupo de contraste en la tarea compensatoria. En este caso, parecería lógico suponer que se ha logrado el objetivo, y que de hecho podemos comprobar la hipótesis, inicial. Sin embargo, esto sería aventurado y supondría confundir la hipótesis conceptual con la experimental. A fin de comprobar la hipótesis, necesitamos conocer si existe un efecto techo (ver Fig. 5a) en la tarea necesitamos conocer si existe un efecto techo (ver fig. 5a) en la tarea compensatoria para el grupo de contraste. Caso de existir este efecto techo necesita- 
FIGURA 5
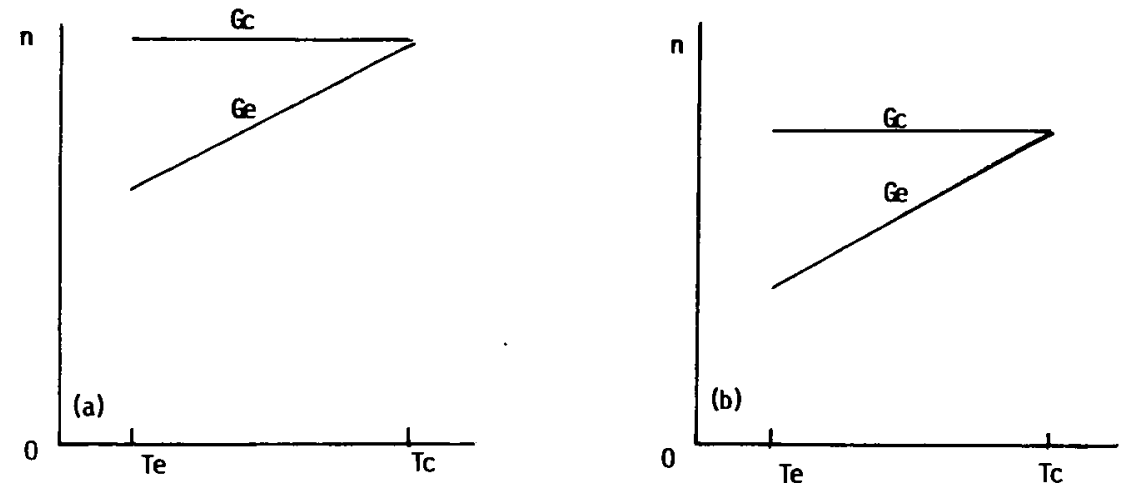

(a) Efecto techo; (b) la hipótesis gana consistencia

mos rectificar la tarea compensatoria. Si no.se presenta el efecto, la hipótesis gana consistencia y podremos decir que se ha comprobado (ver fig. 5b)

Una variante en los resultados puede ser que los sujetos del grupo experimental mejoren en la tarea compensatoria, que además los sujetos del grupo de contraste no mejoren en la tarea compensatoria, pero que sin embargo, la mejora del grupo experimental en la tarea compensatoria no sea suficiente para equipararse al grupo de contrarse (ver Fig. 6). En estos casos, la interpretación de los resultados se dificulta considerablemente ya que dichos resultados pueden deberse a que la modificación incorporada en la tarea compensatoria no es suficiente para activar adecuadamente la operación relevante en los sujetos del grupo experimental. Esta posible interpretación es importante, ya que nos sugiere que la modificación de carácter catalítico dirigida a problemas de mera propensión en la no utilizáción de un mecanismo no es suficiente, y podemos estar ante un problema de capacidad; o bien puede existir alguna fuente adicional de diferencias en la tarea compensatoria. Esto nos llevaría a analizar más en profundidad dicha tarea; puede existir algún artificio por un fallo en proporcionar equivalencia de condiciones en los dos grupos. Para estos problemas, se sugiere registros cualitativos del momento de administración de las tareas.

Figura 6

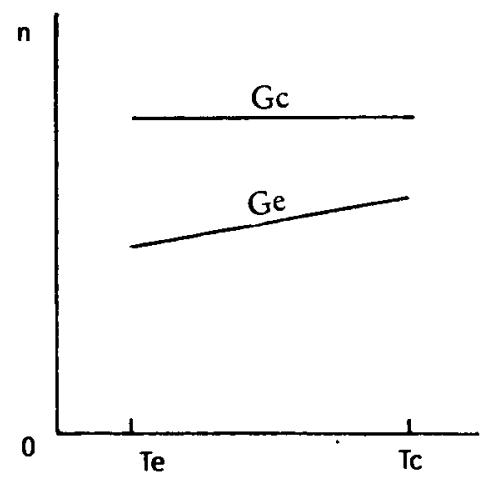

La Tarea Compensatoria no es útil para equiparar el rendimiento de los grupos. Posiblemente problema de capacidad, ofuente adicional de diferencias en la tarea compensatoria 


\section{4}

Otra variante puede resultar en el caso de que los sujetos del grupo experimental mejoren en la tarea compensatoria, pero que también los sujetos del grupo de contraste mejoren en dicha tarea. Entonces nos podemos encontrar con dos resultados; cuando la mejora del grupo de contraste sea superior a la del grupo experimental en la tarea compensatoria (Fig. 7a) y cuando esta no es superior (Fig. $7 \mathrm{~b}$ ). En el primero de los casos, la posible interpretación es que la tarea compensatoria activa alguna operación o mecanismo eficaz que no se utiliza espontáneamente por ambos grupos exigiendo así un análisis más detallado de la tarea compensatoria. En el segundo caso, la posible interpretación es que no todos los sujetos del grupo de contraste utilicen la operación activada por la tarea compensatoria, lo que nos lleva a necesitar revisar nuestros criterios de selección de grupos especialmente en el caso de este grupo de sujetos.

\section{Figura 7}

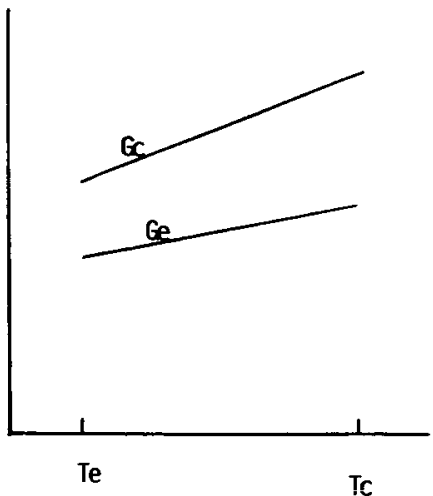

(a)

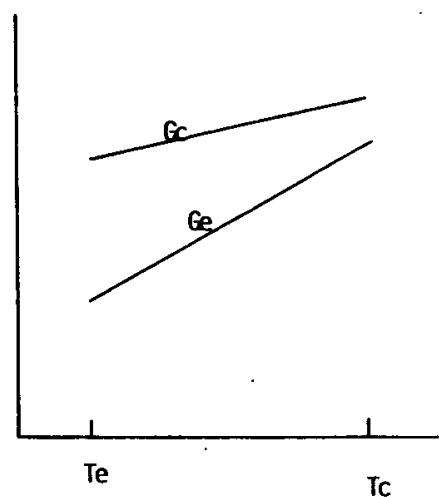

(b)

(a) La Tarea compensatoria activa alguna operación o mecanismo eficaz que no se utiliza espontáneamente por ambos grupos. (b) No todos los sujetos del grupo de contraste utilizan la operación activada por la tarea compensatoria.

Otro resultado es que los sujetos del grupo experimental no mejoren en la tarea compensatoria. Aquí nos podemos encontrar con dos casos. Uno en el caso en el que el grupo de contraste mejore en la tarea compensatoria (ver Fig. 8a). En este caso una posible interpretación es que la tarea compensatoria produzca un efecto "suelo", es decir, que tenga un nivel tan alto para el grupo experimental que la mayor parte de sus puntuaciones se hallen cercanas al nivel cero de ejecución en la tarea. El otro caso se presenta cuando el grupo de contraste no mejore en la tarea compensatoria (ver Fig. 8b). Aquí las posibles interpretaciones podrían ser: a) que la tarea compensatoria no esté directamente relacionada con el mecanismo responsable de las diferencias observadas en la tarea estándar; b) que no se halla logrado la equivalencia en las condiciones de tratamiento.

Subestrategia de entrenamiento:

En términos generales, podríamos decir que esta subestrategia de investigación es muy similar en sus procedimientos e interpretaciones a la de interacción de grupos $\mathrm{x}$ tareas. Procedimentalmente, la diferencia estriba en que, mientras que en la estrategia comentada en el apartado anterior, el in- 


\section{5}

FIGURA 8

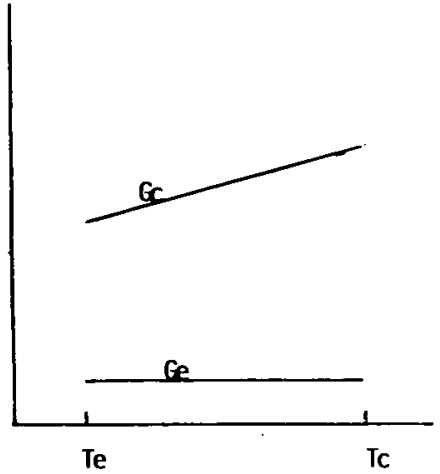

(a)

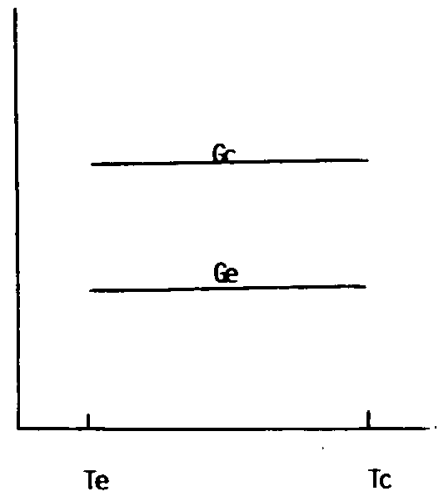

(b)

(a) LaTarea compensatoria produce un efecto suelo. (b) La Tarea compensatoria puede no estar relacionada con el mecanismo responsable de las diferencias, o bien no se ha logrado la equivalencia de tratamiento

vestigador necesita diseñar cuidadosamente la tarea compensatoria, en ésta se necesita un cuidado extremo en el diseño del programa de entrenamiento del grupo objeto de estudio. En terminología de Rohwer (1976), podríamos decir que en las estrategias GxT, la manipulación de variables es fundamentalmente catalitica, mientras que en la estrategia de entrenamiento, la manipulación es fundamentalmente protésica.

El entrenamiento de grupos de nivel diferencial de ejecución, como estrategia en Psicología Diferencial Cognoscitiva, se fundamenta en unos supuestos argumentales similares a aquellos que rigen la simulación por ordenador. Comprendiendo que se requiere para proceder correctamente en un dominio de conocimiento dado, debemos ser capaces de diseñar un programa que, implementado, el ordenador lo lleve a cabo. Por su parte, comprendiendo qué se requiere para proceder correctamente en un dominio de conocimiento dado, debemos ser capaces de prepara un programa de entrenamiento de tales características que, aplicado a sujetos de bajo rendimiento, estos puedan proceder adecuadamente. Por tanto, de la misma manera que para diseñar un programa de simulación capaz de resolver problemas, en el diseño de un programa de entrenamiento de sujetos con rendimiento diferencial necesitamos ser extremadamente explícitos con los pasos que implica una ejecución correcta (Campinone, Brown y Ferrara, 1982).

El diseño básico de la estrategia es el de Test-Entrenamiento-Test unido a un método iterativo de comprobación y/o eliminación de alternativas. Es decir, dado un rendimiento diferencial en un dominio cognitivo representado por la tarea $X$, se explicitan los componentes necesarios para su ejecución correcta, mediante un análisis teórico de la misma, siendo éstos, por ejemplo, $(a b c)$. En el siguiente paso se elabora la hipótesis diferencial asignando a uno de los componentes, por ejemplo el $a$, como fuente del rendimiento diferencial. Se elabora una medida independiente del rendimiento que evalúe el componente que se desea entrenar. Se evalúa a los sujetos; se entrena a los grupos seleccionados a tal fin en el componente a y se obser- 
va el resultado del mismo mediante una evaluación-post. Si bien para un análisis pormenorizado de los posibles resultados puede consultarse el apartado anterior, aludiremos aquí a algunos que nos sirven para ilustrar el método iterativo.

En el caso que el grupo de bajo rendimiento en la tarea $X$ mejore su ejecución de tal modo que se eliminen las diferencias con los de rendimiento normal en dicha tarea y que el grupo de rendimiento normal no mejore significativamente en la tarea; por un lado a) se refuerza el análisis teórico de la tarea y b) gana soporte la hipótesis diferencial sobre la fuente del bajo rendimiento. En este caso, el método iterativo consistiría en volver a repetir el procedimiento con el resto de los componentes de la tarea esperando no encontrar mejoras. Es necesario hacer hincapié en la no mejora del grupo de rendimiento normal ya que, es de suponer que son eficaces desde un principio en a y es de esperar que el entrenamiento no sea eficaz para este grupo. Caso de no ser así, habría que hipotetizar una fuente adicional de diferencias que sería necesario estudiar.

En el caso contrario, podríamos concluir que a no es una fuente de diferencias, es decir el grupo de bajo rendimiento no mejora en la tarea $X$ y procederíamos, mediante el método iterativo, con el mismo diseño de estrategia con el componente $b$, y así sucesivamente. En el caso de que, una vez comprobados los diversos componentes teóricamente responsables de la ejecución eficaz, los sujetos de bajo rendimiento siguieran exhibiendo un rendimiento diferencial, tendríamos que concluir que, o bien el análisis teórico de la tarea es incompleto - cosa que sería enormemente positiva- y que existen fuentes adicionales de diferencias; o bien que nos encontramos ante un componente estructural del sistema cognitivo altamente resistente a la modificación -algo también positivo- Esta última conclusión puede aislarse de la primera en la medida que dispongamos de una medición de los componentes que sea independiente del rendimiento en la tarea, y aún así, resulta difícil decidir si la dificultad de producir mejora en un componente mediante manipulación es debida a la naturaleza estructural del componente o a la dificultad de encontrar un procedimiento adecuado para su entrenamiento. Pese a esta dificultad, el simple hecho de comprobar que la adquisición de un componente es resistente a los medios de entrenamiento habituales, abre al investigador un excitante campo de trabajo sobre los mecanismos de adquisición del mismo.

Este tipo de investigación presenta considerables problemas metodológicos derivados de la naturaleza y puesta en práctica de la misma (Cole y Means, 1981). En cuanto al primer aspecto, podemos encontrarnos con que la multiplicidad de factores que intervienen en el proceso de entrenamiento - consignas, tiempo, tipo de instrucción, etc.,- dificultan la delimitación de cuál de entre estos es el responsable de la mejora que se espera lograr. También podemos encontrarnos con diferencias intragrupos no controladas, o bien que distintos sujetos de un mismo grupo utilicen procesos o estrategias diferentes aunque lleguen a un mismo resultado.

La mayor parte de los autores hacen hincapié en la necesidad de obtener una evaluación del proceso o estrategia hipotetizado como fuente de diferencias que sea independiente del rendimiento en la tarea. De esta manera podríamos paliar tres problemas importantes como son, a) el sesgo en la distribución del proceso o estrategia, ya que permitiría conocer en qué 
medida el componente es de uso común en el grupo de rendimiento alto e infrecuente en los de bajo rendimiento; b) el enmascaramiento de la eficacia del entrenamiento, ya que podemos estimar en qué grado afecta a la utilización del componente en cuestión, y c) la incoherenccia de datos controlando casos como el de puntuaciones deficitarias en grupos de sujetos de alto rendimiento.

Otro problema derivado de esta estrategia, y éste de difícil solución, es la mortandad estadistica, es decir, debido a la duración por lo general larga de estas investigaciones, puede existir abandono significativo de aquellos sujetos que encuentren más dificultades en el entrenamiento de manera que permanecen en la investigación los más aptos o motivados produciendo un incremento artificial de los resultados finales. Si bien este problema es de muy difícil solución, el equipo de investigación debería consignar este hecho cuando así se produzca y ofrecer un informe de las caracaterísticas de dichos sujetos asi como de las posibles causas de su abandono.

Por último debemos señalar que muchas investigaciones que utilizan dos grupos, normal y bajo rendimiento cognitivo, realizan entrenamiento solamente en el de bajo rendimiento bien por problemas de tiempo y/o dinero, o bien porque se considera suficiente el rendimiento del grupo normal. Esta práctica es un error ya que, como hemos comentado, el grupo de rendimiento normal puede obtener mejoras que afectan a la interpretación de los datos, amén de desperdiciar una fuente importante de confirmación. El ideal es utilizar cuatro grupos: rendimiento normal con entrenamiento, rendimiento normal sin entrenamiento; bajo rendimiento sin entrenamiento, y bajo rendimiento con entrenamiento. De esta manera, el grupo de bajo rendimiento sin entrenamiento sirve de control al grupo de bajo rendimiento con entrenamiento ejerciendo un efecto de control sobre posibles factores extraños que pudieran haber incrementado artificialmente el rendimiento. De igual forma, el grupo de rendimiento normal sin entrenamiento asegura que, caso de haber mejoras en el grupo de bajo rendimiento con entrenamiento, estas se deben a efectos del mismo, informándonos de posibles fuentes adicionales de diferencias.

Subestrategias de comparación de grupos con tareas de modelos explícitos

En este tipo de subestrategias se parte de una teoría cognitiva, observación empírica, o inducción lógica, para desarrollar un modelo sobre la forma en que los sujetos más eficaces resuelven una tarea específica, así como modelos alternativos de dichas tareas que representen la ejecución de la misma por sujetos menos eficaces. Una vez que se han generado dichos modelos hipotéticos sobre las ejecuciones, se compara el resultado de cada modelo - derivado a partir de deducción lógica, matemática o de simulación por ordenador- con la ejecución de cada grupo de sujetos en la tarea propuesta. Por último, el investigador evalúa la proporción de sujetos que, en cada grupo, muestran una propensión a emplear un modelo de ejecución de la tarea dada, lo que le permite interpretar las diferencias cognitivas en función de las diferencias entre modelos.

\section{Análisis lógico-racional de tarea:}

Este tipo de análisis especifica explícitamente la secuencia de procesos que realiza el individuo en la ejecución de una tarea. Esto permite elaborar 
diferentes modelos respecto a una misma tarea, como forma de dar cuenta de las diferencias individuales. El análisis lógoco-racional se podría describir mediante los siguientes puntos:

a) Variar sistemáticamente la estructura de la tarea en las dimensiones hipotéticas revelantes, para dar lugar a un conjunto de problemas.

b) Generar el patrón de respuestas esperado (por ejemplo mediante la creación de diagramas de flujo explicativos).

c) Comparar este patrón esperado con las respuestas de los sujetos, a través de algún criterio para decidir cuándo se verifica nuestro patrón hipotético generado.

Una vez analizada la tarea, se establecen una serie de variaciones para determinar la estrategia utilizada por cada sujeto. Por ejemplo, Siegler (1976) sleccionó problemas de equilibrio de balanza para producir diferentes patrones de rendimiento según las diferentes reglas utilizadas por cada niño. Seleccionó seis tipos de problemas, de forma que después de varios ensayos, la ejecución del niño reflejase la regla que estaba utilizando. Siegler (op. cit.) utilizó el método de evaluación de reglas. El objetivo de su investigación fue descubrir cómo niños y adolescentes resolvían los problemas de la balanza (Fig. 9). Podía variarse el número de pesos y la distancia al centro de la balanza. El sujeto debía decidir hacia dónde se inclinaría la balanza (una vez manipulados los pesos y las distancias).

\section{FIGURA 9}

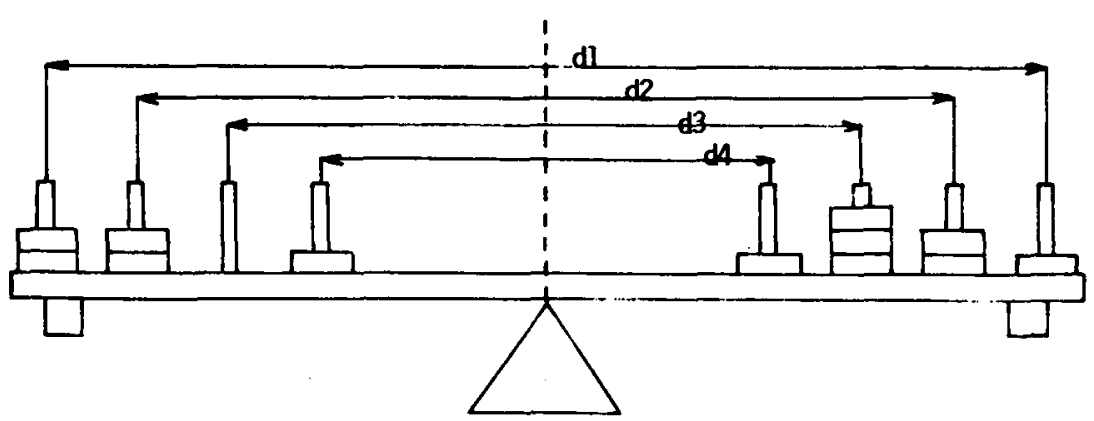

Balanza de la investigación de Siegler (1976). Las variaciones experimentales se producen en los pesos $y$ en las distancias (i.e. $d 1, d 2, d 3$ y d4) cuyas diversas combinaciones originaban un espectro considerable de problemas

Basándose en un análisis de la tarea y en investigaciones previas Siegler propuso cuatro estrategias, o reglas, que supuestamente usarían los sujetos en la resolución de este problema. La Regla I supone prestar atención únicamente a la cantidad de pesas que hay en cada brazo de la balanza, ignorando las distancias respectivas entre las pesas y el soporte. La Regla II toma en cuenta la distancia, pero sólo cuando los dos brazos tienen la misma cantidad de pesas. Según la Regla III se tiene en cuenta peso y distancia, pero cuando un brazo tiene más pesas y en el otro las pesas están más lejos del pie, el niño no resuelve el problema. Por úlimo, la Regla IV (solución madura) incorpora una estrategia para resolver el problema que se presentaba en la regla anterior: multiplicar distancia por peso para cada es- 
taca, sumar los productos obtenidos de cada lado y comparar el resultado de la suma de la derecha con el de la izquierda; el sujeto que utiliza esta Regla predice que descenderá el lado cuya suma de productos sea mayor.

Para valorar la adecuación de estas estrategias, Siegler creó subtipos de problemas diferentes variando la estructura, o los parámetros básicos, de la tarea. Evaluó a 120 sujetos de 5 a 17 años, en 30 problemas resultado de esa variación. Las respuestas de cada individuo secompararon con las predichas por la regla. El criterio se daba si se predecían correctamente 26 de las 30 respuestas. Se encontró que 107 de los 120 sujetos utilizaron un patrón correspondiente a una de las cuatro reglas.

Una de las características esenciales de la investigación basada en modelos es el análisis detallado de la tarea describiendo explícitamente los sistemas de reglas aplicadas en las etapas de desarollo. Este análisis se acompaña de una prueba estructurada de manera que se ponga de manifiesto la estrategia utilizada por cada individuo. Este enfoque permite estudiar las diferencias entre individuos que usan diferentes sistemas de reglas, así como las interacciones entre la edad y el modo en que se aplica determinada regla. Permite indicar en qué consisten las diferencias de ejecución.

No obstante, este tipo de análisis racional tiene una serie de problemas. En primer lugar, se necesitan muchos problemas para mostrar concluyentemente en un patrón de respuestas es consistente con una regla, y no con otras, y para que el patrón difiera significativamente del producido por respuestas aleatorias (esta limitación es menos importante cuando el problema es de solución rápida y se prueban pocos modelos). En segundo lugar, si se analizan los patrones de respuesta, los individuos deben responder de acuerdo a una estrategia en casi todos los problemas. Por último, la evaluación de reglas no puede discriminar entre estrategias funcionalmente equivalentes, respecto a los patrones de respuesta. En algunos casos, estrategias radicalmente diferentes pueden dar lugar a patrones de respuesta idénticos (Simon, 1976). En tales casos, se requerirán métodos más sensibles.

Análisis de simulación por ordenador y análisis matemático de tarea:

Tanto en las subestrategias de simulación por ordenador, como aquellas cuyas tareas obedecen a análisis explícitos de tareas mediante procedimientos de simulación matemática, no realizaremos un análisis detallado de sus fundamentos ya que esto ha sido tratado en los apartados correspondientes. Unicamente presentaremos la forma en que podrían utilizarse para los estudios la comparación de grupos.

\section{a. Análisis mediante procedimientos de simulación por ordenador}

La simulación por ordenador pretende ser un reflejo de la actividad del sujeto humano ante una tarea. En su momento comentamos la relación que guarda la simulación con la IA, y los distintos tipos de simulación que podíamos encontrar. En esta subestrategia consideramos las posibilidades de la comparación de grupos, con tareas que tienen un modelo explícito obtenido mediante simulación por ordenador. De esta forma, pasaremos por alto la elaboración de un programa para ir a las especificaciones en esa subestrategia. En primer lugar, se toma una tarea, con un análisis explícito 
de simulación, para su ejecución correcta; a continuación se eligen grupos de rendimiento normal, y bajo rendimiento, o bien grupos de expertos en diversos campos, caso que el interés sea comparar las diferencias existentes entre expertos. Posteriormente se comprueba el ajuste del modelo de simulación con el grupo de expertos calibrando su bondad de ajuste, y se analiza mediante alguna técnica (p.e. análisis de protocolos) las ejecuciones de la tarea por el resto de los grupos elegidos. A partir de los datos de los grupos de bajo rendimiento, se diseñan programas que simulen estos rendimientos. Se comparan los programas diseñados con los datos obtenidos, hallando la bondad de ajuste, y, por último, se comparan los distintos modelos de simulación interpretando los resultados.

La ventaja de este tipo de subestrategia consiste en que se comparan «teorías» contrastadas con los modelos de distintas modalidades de ejecución o rendimiento. De esta manera, no estamos hallando simplemente, como en las estrategias de modelos implícitos, un déficit o un componente en el que falla uno de los grupos de sujetos, sino que nos permite averiguar y/o describir una teoría o modalidad del rendimiento diferencial en los casos de sujetos con bajo rendimiento. Un ejemplo de este tipo de investigaciones puede ser el llevado a cabo por Young (1978) en el cual se planteaban tres modelos de simulación de distintas modalidades de ejecución sobre tareas de seriación.

La teoría de Young (1978) sobre la seriación de los tamaños combina una metodología computacional con una experimental. Pretende demostrar que el comportamiento de este tipo, a cualquier edad, puede ser descrito por medio de Sistemas de Producción (SPs) específicos. El paso de un estadio a otro se produciría de forma progresiva por medio de la adición de una o varias Reglas de Producción (RPs) al sistema original. Las reglas que se van añadiendo al sistema son psicológicamente pausibles.

Para contrastar su teoría, Young recurre a la observación. Va emparejando los SPs a los comportamientos de los niños. Cuando se produce un mal emparejamiento, se busca la causa en el sistema de producción, y se intenta lograr la regla que produzca el comportamiento deseado. Se detallan rasgos como la elección de un bloque específico en lugar de otro, el movimiento de la mano hacia un bloque que después no se elige, etc. Aunque los rasgos generales de dos conductas sean los mismos, los detalles pueden darnos pistas muy importantes sobre las diferencias.

Los SPs son idiosincrásicos, en el sentido de que cada niño posee un conjunto diferente de RPs que pueden ser descritas (Fig. 10). Esto supone resaltar la riqueza de las diferencias individuales de forma mucho más marcada que las medidas utilizadas habitualmente en la psicología diferencial, basadas en procedimientos estadísticos.

Es interesante observar el aumento en la cantidad de RPs a medida que el niño aprende a seriar. Los predicados están enunciados de forma intencional, es decir, se refieren a aquella información almacenada en la MCP, que representa el conocimiento del sistema de cuáles son las condiciones satisfechas, y cuáles no. Existen diferencias intra-individuales en el comportamiento de seriación - a veces un niño coge un bloque depués de pensarlo un rato, otras veces coge uno al azar, otras veces coge el que está al lado del que en un principio ha elegido, etc.- - En este sentido la regla S2 es fundamental: es quizá la clave del comportamiento de seriación. Es muy 
FIGURA 10

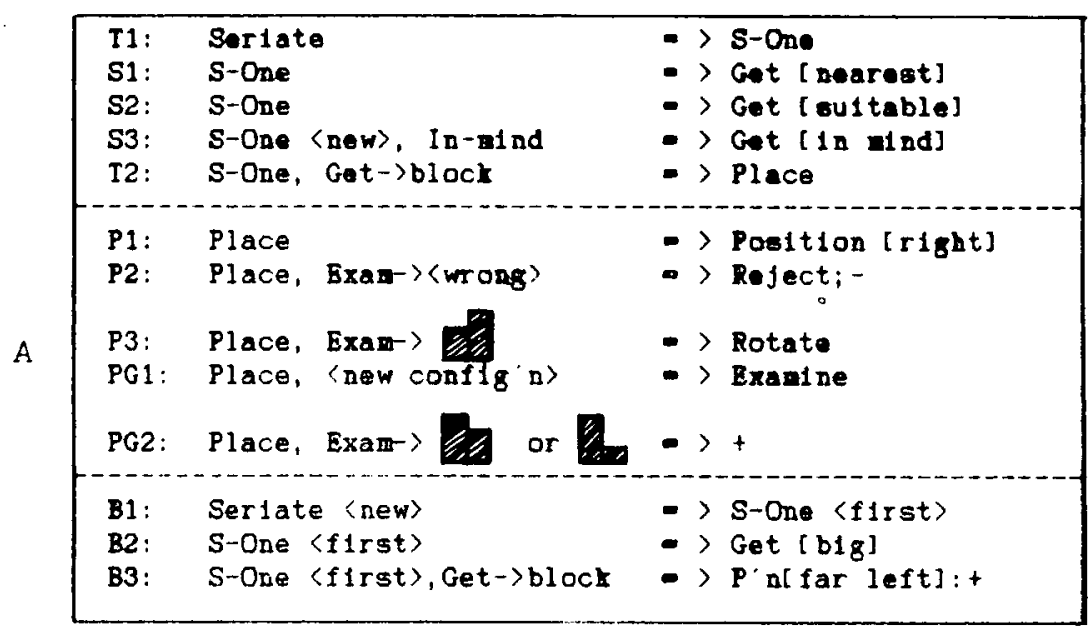

\begin{tabular}{|c|c|c|}
\hline $\begin{array}{l}\text { T1: } \\
\text { S1: } \\
\text { T2: }\end{array}$ & $\begin{array}{l}\text { Ser1ate } \\
\text { S-One } \\
\text { S-One, Get->block }\end{array}$ & $\begin{array}{l}\text {-> S-One } \\
->\text { Get [ next nearest] } \\
->\text { Place }\end{array}$ \\
\hline $\begin{array}{l}\text { P1: } \\
\text { PG1: } \\
\text { PG2: } \\
\text { PG3: }\end{array}$ & $\begin{array}{l}\text { Place } \\
\text { Place, 〈new configuration> } \\
\text { Place, Examine-> } \\
\text { Place, Examine-> }\end{array}$ & $\begin{array}{l}\text {->Position [right] } \\
->\text { Examide } \\
->+ \\
->\text { Switch }\end{array}$ \\
\hline $\begin{array}{l}\text { B1: } \\
\text { B2: } \\
\text { B3: }\end{array}$ & 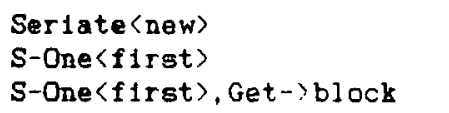 & $\begin{array}{l}\Rightarrow \text { S-One }\{\text { f1rst }> \\
\Rightarrow \text { Get [b1ggegt] } \\
\Rightarrow \text { Pos n[far left]: }\end{array}$ \\
\hline
\end{tabular}

\begin{tabular}{|c|c|c|}
\hline $\begin{array}{l}\text { I1': } \\
\text { I2': }\end{array}$ & $\begin{array}{l}\text { Sertate } \\
\text { Serlate, Get->block }\end{array}$ & $\begin{array}{l}\Rightarrow \text { Get[ b1 g8est }] \\
\Rightarrow \text { Posttion[ } r \text { ght }]\end{array}$ \\
\hline B3 $^{\prime}:$ & Serlate $\langle$ new $\rangle$, Get->block & - Position[far left] \\
\hline
\end{tabular}

Modelos de simulación de distintas modalidades de ejecución en tareas de seriación, A) proceso de un niño en su último estadio de seriación; B) proceso de un niño menos avanzado, y C) proceso de un niño en el primer estadio de seriación. (Tomado de Young, 1978) 
posible que esta regla, y la posibilidad de que su adquisición en distintos grados, sea generadora de las diferencias individuales.

Las reglas que componen los SPs que estudia Young están agrupadas en:

1. Relacionadas con la estructura general de la acción.

2. Selección de los bloques a añadir

3. Evaluación de la parte de la seriación ya construida

4. Correcciones de los bloques incorrectos.

Young propone un espacio tridimensional de habilidades de seriación en el que se pueda representar a cualquier niño que atraviese este período. El punto en el que se sitúe cada niño indicará las características de la ejecución del niño, los problemas que puede y no puede resolver, y la naturaleza de sus errores. Cada estadio de seriación se corresponde con una región concreta de dicho espacio tridimensional.

Una modalidad de aplicación de las estrategias de comparación de grupos en IA puede encontrarse en las investigaciones de refinamiento de sistemas expertos. Por ejemplo, Johnson et. al., (1981) crearon un sisema experto, DIAGNOSER, capaz de generar diagnósticos médicos en tareas específicas. A fin de proporcionar más poder a dicho sistema realizaron varios experimentos con tres grupos: uno de expertos diagnosticadores, sujetos en período de formación, y estudiantes de medicina. La finalidad fue alcanzar una mayor comprensión de los diagnósticos incorrectos.

\section{b. Análisis mediante modelos matemáticos}

Al igual que en el apartado anterior, no comentaremos los fundamentos de los modelos matemáticos, sino que nos remitimos al partado correspondiente a las estrategias de simulación matemática, para una mayor profundización.

Tal y como comentamos, estos modelos matemáticos pretenden simular el comportamiento ante una tarea, a través de una función matemática en la que se asignan valores numéricos a las variables. En estas subestrategias de comparación de grupos:

1. Se elige la tarea a través de la cual se pretende analizar el rendimiento diferencial de los grupos.

2. A partir de un modelo teórico de la tarea, se establece una función matemática que simule su ejecución correcta.

3. Se eligen los grupos de alto y bajo rendimiento, a los que se somete a las tareas tomándose los datos de los sujetos de alto rendimiento para calibrar los parámetros de la función matemática observándose la bondad de ajuste.

4. A partir de los resultados obtenidos por los sujetos de rendimiento diferencial o bajo rendimiento, se corrigen los parámetros del modelo de tal manera que permitan alternativas en la ejecución de la tarea.

5. Se observa la bondad de ajuste entre los grupos de bajo rendimiento con la función matemática de simulación.

6. Se interpretan los resultados.

Al igual que en las subestrategias de comparación de grupos mediante simulación por ordenador, la ventaja que se obtiene de estos modelos de 
función matemática es que no solamente se conoce en qué parámetro no ejecutan los sujetos adecuadamente, siendo éste responsable de las diferencias, sino que se conoce cuál es la alternativa de ejecución deficitaria.

Un ejemplo de investigación con este tipo de subestrategias es la llevada a cabo por Gutowski y Checile (1987). El problema que abordaron se enmarca dentro de los estudios sobre codificación, almacenamiento y recuperación como componentes de diferenciación cognitiva, desde la perspectiva de la expliación multiprocesual. Dicho problema lo aplican a memoria con tareas de memoria asociativa a corto y largo plazo. Para abordar el tema utilizan la comparación de grupos extremos con sujetos retrasados y sujetos normales.

El procedimiento elegido tenía precedentes, en el marco de trabajo mencionado, con las técnicas desarrolladas por Checile y Meyer (1976; citado en Gutowski y Checile, 1987). Bajo los supuestos de estas técnicas pasaron pruebas de memoria asociativa en intervalos de retención mínimo, y en intervalos más extensos, entremezclando aleatoriamente los ensayos de recuerdo con los ensayos de reconocimiento si-no; a su vez, en los ensayos de reconocimiento se aplicaba una escala para medir el grado de confianza de los sujetos. De acuerdo con ésta tarea general se computan tres respuestas de interés para cada sujeto: $\theta \mathrm{c}=$ proporción de tiempo en la que se codifica la información; $\theta \mathrm{a}=$ proporción de tiempo en el que se almacena la información una vez codificada; $\theta \mathrm{r}=$ proporción de tiempo en recuperar la información, una vez codificada y almacenada.

Para explicar las respuestas de los sujetos, se formularon un conjunto de ecuaciones que pretendían simular el comportamiento diferencial de los sujetos ante estas condiciones. Dichas ecuaciones se presentan a continuación:

La proporción resultante del déficit diferencial de retención debido al almacenamiento sería:

$$
\mathrm{k} \alpha_{\mathrm{a}}(\mathrm{x})=\left[1+\alpha_{\mathrm{a}}(\mathrm{x}) \alpha_{\mathrm{c}}(\mathrm{x})+\alpha_{\mathrm{r}}(\mathrm{x}) / \alpha_{\mathrm{c}}(\mathrm{x}) \alpha_{\mathrm{r}}(\mathrm{x})\right]^{-1}
$$

La proporción resultante del déficit diferencial de retención debido a la recuperación sería:

$$
\mathrm{k} \alpha_{\mathrm{r}}(\mathrm{x})=\left[1+\alpha_{\mathrm{r}}(\mathrm{x}) \alpha_{\mathrm{c}}(\mathrm{x})+\alpha_{\mathrm{a}}(\mathrm{x}) / \alpha_{\mathrm{c}}(\mathrm{x}) \alpha_{\mathrm{a}}(\mathrm{x})\right]^{-1}
$$

Finalmente, la proporción resultante del déficit diferencial de retención debido a la codificación sería:

$$
\mathrm{k} \alpha_{\mathrm{a}}(\mathrm{x})=1-\mathrm{k} \alpha_{\mathrm{a}}(\mathrm{x})-\mathrm{k} \alpha_{\mathrm{r}}(\mathrm{x})
$$

siendo $\alpha_{c}$ la eficiencia relativa de la codificación entre retrasados y normales, es decir, la proporción entre los valores $\theta c$ entre los grupos extremos; $\alpha_{\mathrm{a}}(\mathrm{x})$ y $+_{\mathrm{r}}(\mathrm{x})$ las eficiencias de almacenamiento y recuperación en un intervalo de retención dado, siendo $(x)$ dicho intervalo de retención debido. Para determinar la proporción del déficit diferencial de retención a cada proceso de memoria se define una medida de la proporción o eficiencia relativa de un proceso para la codificación. La proporción no-normalizada de la eficiencia relativa de la codificación para sí mismo es 1; no obstante, para el almacenamiento es $\alpha_{\mathrm{c}}(\mathrm{x}) / \mathrm{am}(\mathrm{x})$. La proporción no normalizada de 
la eficiencia relativa de la codificación para la recuperación es $\alpha_{c}(x) / \alpha_{r}(x)$. $\mathrm{K}$ sería la constante de normalización para transformar las eficiencias relativas en proporciones del déficit diferencial total de retención, y dicha $\mathrm{K}$ es múltiplo de la proporción normalizada de las eficiencias relativas y asegura que la suma de las tres proporciones es 1.

\section{La estrategia de contrastación mediante simulación diferencial}

Este tipo de estrategias comentadas por Rohwer (1976) son quizá una de las teóricamente más interesantes al cumplir las condiciones de una investigación puramente experimental; es decir, los sujetos pueden ser asignados al azar a las condiciones del experimento, y se manipula la variable independiente. No obstante, decimos que esta es una estrategia de contrastación debido fundamentalmente a la dificultad de inhibir un proceso cognitivo y estar seguros de que las acciones que se efectúan para producir dicha inhibición no afectan a otros procesos o componentes de estrategia para la resolución de la tarea. Sin embargo, no descartamos la posibilidad de que puedan surgir desarrollos tecnológicos que nos permitan dicha intervención. Por el momento, contemplamos esta estrategia como una forma de contrastar los resultados obtenidos en las anteriores estrategias de comparación de grupos.

El procedimiento es sencillo, y se guía por las caracaterísticas de cualquier diseño experimental. Aquí lo expondremos sucintamente:

1. Elegir la tarea objeto de estudio.

2. Elaborar una hipótesis diferencial sobre el proceso o componente responsable de dicha diferenciación. Esta hipótesis debe ser avalada por estudios anteriores de estrategias de comparación de grupos, y deben de tomarse los datos que luego permitan la contrastación.

3. Explicitar, de forma clara, la forma en que se va a inhibir el proceso o componente de estrategia que se considera responsable de un bajo rendimiento.

4. Asignar la muestra de los sujetos al azar a las condiciones de intervención (inhibición del proceso), y no intervención.

5. Evaluar a los sujetos en la ejecución de la tarea.

6. Aplicar el procedimiento de inhibición al grupo correspondiente.

7. Evaluar de nuevo a ambos grupos, respecto a su ejecución en la tarea, y observar los resultados obtenidos.

Lógicamente lo que es de esperar es que los sujetos sometidos al procedimiento de inhibición presenten una disminución de su rendimiento equivalente a la que supondría el haber utilizado un grupo de bajo rendimiento. Como es de suponer, y antes mencionábamos, para ello es conveniente tener datos de comparación de grupos de alto y bajo rendimiento.

El proceso, al igual que comentábamos en las subestrategias de entrenamiento, podría convertirse en iterativo, es decir, ir pasando de componente a componente hasta que se obtuviera el rendimiento deficitario buscado. Una variante de esta tarea es emplear conjuntamente este tipo de procedimiento con el de comparación de grupos de alto y bajo rendimiento, con lo que podríamos comparar de forma directa las curvas de rendimiento entre el grupo de los sujetos a los que se les aplica la inhibición, y las evaluaciones de los sujetos de bajo rendimiento. 
Tal y como advertíamos en el entrenamiento de grupos, es de enorme importancia contar con una medida del proceso o componente independiente del proceso de la tarea. De no ser así, no sabríamos en qué medida la inhibición en el componente específico queda enmascarada por el procedimiento para inhibirla, u otros factores colaterales.

Esta estrategia, desde el punto de vista teórico, podría resultar de gran utilidad en la medida en que pudiéramos contar con medidas independientes de los diversos procesos o componentes de la tarea, y pudieran establecerse análisis múltiples de los resultados, o de las evaluaciones en dichos componentes, ya que contariamos con indicadores, cuando menos heurísticos, del influjo que pudiera tener un proceso o componente inhibido, es decir, su ausencia o su dificultad de acceso en otros componentes implicados en la tarea; con lo que el análisis del rendimiento diferencial, es decir, el cómo se diferencian los sujetos, quedaría ampliamente reforzado.

\section{PROBLEMAS ASOCIADOS}

Diversos investigadores, a lo largo de varias décadas, han enfatizado no solamente las ventajas que proporcionaban los estudios de comparación de grupos, sino también los problemas metodológicos ligados a este tipo de investigaciones que pueden afectar seriamente a la interpretación de los resultados.

Cuando comparamos dos o más grupos con una o más tareas o modificaciones de tareas, esperamos que las variaciones en los datos puedan corresponderse con las fuentes de variación hipotetizadas. Sin embargo, dicha interpretación puede verse enturbiada por problemas asociados: a) bien a las pruebas o tareas; b) bien a la selección de los grupos y su emparejamiento. Es decir, desde el punto de vista de las tareas cualquier cambio en la diferente ejecución de los dos grupos depende, no sólo de sus respuestas a la variable, per se, sino también, por una parte, de las características psicométricas de las tareas, que pueden tener poco que ver con lo que se pretende medir; y por otra, al tipo de relación existente entre la variable cognitiva que se hipotetiza como fuente de diferenciación y los datos obtenidos como resultado de emplear tareas que pretenden medir dicha variable; esto es, entre la hipótesis explicativa y la instrumental. Desde el punto de vista de la selección de los grupos de sujetos, siendo el objetivo de estos estudios atribuir las diferencias a una variable del sujeto, nos encontramos con la dificultad de aislar dicha variable e ignorar otras potencialmente relevantes, a tal fin se suele emplear como procedimiento el emparejamiento de grupos, procedimiento éste no exento de dificultades como veremos.

\section{a) Problemas asociados a las tareas}

Por lo general, cuando comparamos grupos respecto a tareas introduciendo modificaciones que afectan a la precisión, bien confeccionando tareas compensadoras, bien diseñando entrenamientos, etc., esperamos que dichas manipulaciones afecten a la variable dependiente de tal manera que las diferencias iniciales entre grupos se vean eliminadas o al menos reducidas significativamente. Si pretendemos asociar cualquier variación en la ejecución de los grupos de sujetos a un componente de diferenciación, nece- 
sitamos que las pruebas o tareas que lo representan sean sensibles a la ejecución diferencial de los sujetos. Dicha sensibilidad la denominamos comúnmente "capacidad discriminante», y depende de la dificultad y fiabilidad de la prueba. Estas caracaterísticas pueden provocar efectos que hagan que la interpretación de los resultados sea equívoca.

En cuanto a la dificultad de la tarea, los problemas más comunes que nos podemos encontrar con los llamados «efectos suelo" $\mathrm{y}$ «efectos techo".

Cuando se emplea una tarea donde todos, o la gran mayoría de, los items presentan un nivel de dificultad mayor que el nivel de máxima diferenciación entre los grupos, nos contramos que las curvas de ejecución de ambos grupos - alto y bajo rendimiento- por lo regular serán divergentes con respecto a los niveles más elevados de la variable independiente. A este fenómeno se le denomina "efecto suelo». En otras palabras, se produce este efecto cuando la mayoría de los items tienen un nivel de dificultad tan alto que el grupo de peor rendimiento presenta puntuaciones excesivamente bajas (ver Fig. 8a).

Por otro lado, cuando los items de la tarea presentan una dificultad menos que el nivel de máxima diferenciación de grupos, las curvas de ejecución de ambos grupos serán, por lo regular convergentes con respecto a los niveles más elevados de la variable independiente. A este fenómeno se le denomina "efecto techo». Su sentido es que la tarea tiene un nivel de dificultad excesivamente bajo y por tanto, el grupo de rendimiento normal presenta la mayoría de sus puntuaciones cercanas al nivel de dificultad máxima de la tarea (ver Fig. 5a)

Otra característica que afecta a la capacidad discriminante de una prueba es su fiabilidad. La fiabilidad de una prueba está determinada en parte por el nivel de dificultad. Mientras que la dificultad de los ítems. como hemos visto, afecta a la diferencia entre los sujetos más y menos capaces, la fiabilidad de una prueba, por su parte, afecta a las diferencias intraindividuales de los sujetos. En investigaciones de comparación de grupos en las que ha de utilizarse una tarea, o versión de la misma, en dos ocasiones, la fiabilidad es uno de los elementos que más gravemente puede enturbiar los resultados; cuando han de emplearse versiones modificadas de la misma, la tarea se complica mucho más y ha de procederse al emparejamiento de las pruebas, procedimiento éste que conlleva muchos riesgos ya que para tal proceder ha de contarse tanto con la dificultad como con la fiabilidad.

Las pruebas que difieren en dificultad, pero son iguales en fiabilidad, tenderán a no producir la misma diferencia en la puntuación media entre los subgrupos de sujetos de alta y baja puntuación. Manteniendo otros factores constantes, la dificultad promedia de los ítems produce una varianza de test mayor en todo el grupo. Si se asume la equivalencia de las fiabilidades, el test con mayor varianza producirá las mayores diferencias promedio entre subgrupos. No se pueden evitar los efectos de la dificultad en la diferencia en la puntuación media de los grupos limitando la manipulación de una variable al campo promedio de dificultad, utilizando por ejemplo, tareas que produzcan una proporción de error entre 25 y $75 \%$. El campo promedio de dificultad de los ítems producirá una fiabilidad mayor, lo que afectará a la varianza observada del test (Chapman y Chapman, 1985).

El emparejamiento de tarea será significativo sólo si los sujetos de menor capacidad muestran varianza en los ítems más fáciles, y si las tareas es- 
tán emparejadas en los ítems fáciles, y en general en toda la tarea. Los sujetos de alto rendimiento son el grupo de estandarización apropiado. No se deben emparejar las tareas en grupos de sujetos de alto y bajo rendimiento combinados, porque las fiabilidades se verán afectadas por cualquier déficit diferencial debido al escaso rendimiento (se elegirán los ítems menos fiables para la tarea en la que los sujetos de bajo rendimiento tienen el mayor déficit). Las tareas, además, se deben emparejar en su fiabilidad, utilizando por ejemplo el coeficiente alfa. Por último, se debe asegurar que se esté midiendo la misma capacidad en los diferentes niveles de dificultad. Para este menester, el investigador debe analizar los ítems. P.e. debe decidir no utilizar una tarea de definición de palabras en la que los ítems fáciles se refieren a eventos concretos y los ítems más difíciles se refieren a ideas abstractas; el investigador puede analizar factorialmente cada tarea en sujetos normales sólo, y verificar que los mismos factores se presentan en los ítems fáciles y en los difíciles.

Por último nos referiremos al problema de la relación entre la variable cognitiva hipotetizada como fuente de diferenciación y los datos obtenidos por los sujetos en la tarea que pretende representarla. Este problema es especialmente importante en Psicología Diferencial Cognoscitiva ya que el interés fundamental está en la variable cognitiva, inaccesible a la observación directa.

La interpretación de los resultados en investigaciones que utilizan fundamentalmente como variable dependiente la probalidad de respuesta tiene una serie de inconvenientes. Normalmente se asume una función lineal entre los datos y la variable hipotética; esto no es lícito porque podemos encontrarnos con otro tipo de funciones (negativamente acelerada, ogival, etc.) que sean las que realmente se ajusten a la relación. En la medida en que no se considera la naturaleza de esta función, se corre el serio riesgo de sobreinterpretar los datos.

Se podrían definir cinco clases de componentes teóricos, de acuerdo a si las funciones son monotónicas, ogivales, negativamente aceleradas, inversas o lineales. Una interacción es interpetable en términos de la probabilidad de respuesta, si el ordenamiento de los datos de la probabilidad de respuesta, y la forma de la función de proyección que consideramos, específica sin ambigüedades la forma en la que podría darse la interacción, en términos del componente teórico.

Las diversas clases de componentes teóricos, tienen, supuestamente, la capacidad de estar cuantitativamente relacionadas con la probabilidad de respuesta. Dentro de éstas, se pueden encontrar dos subclases:

a) Se suponen especificadas la función y sus parámetros; esto es, dada determinada probabilidad $\mathrm{p}(\mathrm{x})$, se puede determinar el valor numérico de $\mathrm{x}$.

b) Implica ejemplos en los que se conoce o intuye la forma general de la función (p.e. la función es negativamente acelerada), pero por una $u$ otra razón, no está determinada la forma y/o los parámetros de la función - p.e. no se conoce si la función es logarítmica o exponencial-.

Generalmente, se asume que la mayor parte de las variables se distribuyen normalmente. Supuesta una distribución normal subyacente, el componente teórico de interés se mide mediante puntuaciones normalizadas $(\mathrm{z})$. 
Determinadas partes de la función ogival son aceleradas negativas, mientras que otras son aceleradas positivas.

Cuando el componente teórico de interés es el tiempo de ejecución de una operación, generalmente una función inversa de uno u otro tipo proyecta el tiempo en la probabilidad de respuesta. Esto es, cuanto más tiempo requiera realizar determinado proceso, más baja será la probabilidad de realizar adecuadamente la tarea. Cualquier interacción que implique curvas convergentes en los niveles más altos de probabilidad, debe producir una interacción convergente en términos del componente teórico. Las únicas interacciones no interpretables son las que implican curvas divergentes en los niveles más altos de probabilidad.

El punto crucial, según Loftus (1978), es que una VD como la probabilidad de respuesta no es interesante intrínsecamente. Más bien, es sólo interesante por lo que refleja de un componente de determinada teoría. La proyección de la VD al componente teórico puede implicar una transformación de la VD. Si esta transformación no es lineal, entonces se encuentran limitaciones en la interpretación de los datos que suponen la VD original.

\section{b) Emparejamiento de grupos}

Uno de los principales escollos en la investigación por comparación de grupos estriba en la imposibilidad de asignación al azar de los sujetos a las tareas dadas las características de los mismos - retraso mental, etc.-, exceptuando las estrategias de simulación de diferencias. Siendo el objetivo de los estudios de comparación de grupos, atribuir las diferencias a una variable del sujeto, nos encontramos con la dificultad de aislar dicha variable e. ignorar otras potencialmente relevantes. Es decir, nos enfrentamos a la dificultad de descartar n-hipótesis alternativas. Para ello, uno de los procedimientos más utilizados consiste en el emparejamiento de grupos una vez elegido el criterio de selección. Cole y Means (1981) analizan este procedimiento estableciendo dos tipos: a) equiparación de grupos en función de características de los sujetos no estudiadas directamente y que, potencialmente, pudieran ser responsables del efecto que se pretende predecir, y b) equiparación de grupos en función de una capacidad que se supone relacionada con el comportamiento objeto de estudio. Ambos tipos plantean problemas.

El primero de ellos presenta la dificultad de que nunca se puede tener seguridad de haber logrado un emparejamiento suficiente; es más, puede ocurrir que una variable que no haya sido tomada en cuenta pueda resultar crucial para el estudio. Además, es un proceso costoso y, al intentar un equiparamiento en algunas variables, podemos producir una des-equiparación en otra.

El segundo, y quizá uno de los más utilizados en Psicología Diferencial Cognscitiva, es que el emparejar a los grupos en capacidades, como inteligencia, etc., medidas a través de test, presenta problemas asociados a artificios estadísticos. Algunos autores desaconsejan este tipo de emparejamiento (Cole y Means, 1981) a causa, específicamente, de los efectos espúreos relacionados con la regresión estadística que a menúdo se producen. 


\section{CONSIDERACIONES FINALES}

En este artículo hemos presentado la problemática metodológica que presenta la Psicología Diferencial Cognoscitiva como disciplina situada entre la Psicología Diferencial Psicométrica y la Psicología Cognitiva y del Procesamiento de la Información. El interés de esta disciplina consideramos que se centra en averiguar cómo y porqué se diferencian los individuos frente al qué y cuanto planteado por la Psicología Diferencial Psicométrica.

En concreto hemos abordado las estrategias metodológicas de primera generación que tratan de analizar problemas de espectro reducido sin pretensiones de generalidad, lo cual se puede encuadrar dentro de la psicología del procesamiento de la información que pretende elaborar micromodelos de determinados aspectos de la cognición.

La presentación de estas estrategias obedece a una necesidad de llenar un vacío de la disciplina psicológica en nuestro país que consistía en la carencia de un intento sistemático de orientar hacia la metodología necesaria para poder llevar a cabo investigaciones en cognición con las diferencias individuales como asunción de partida. Nuestro deseo es que este artículo sea del interés suficiente como para servir a este fin.

En este artículo no se abordan las estrategias de segunda generación, es decir, aquellas metodologías caracterizadas por un poder descriptivo y explicativo que rebasa las pretensiones de las estrategias de primera generación, por lo que se refiere a la generalidad de las derivaciones teóricas a partir de los resultados obtenidos. Además, estas estrategias de segunda generación pretenden aumentar el alcance de las posibles aplicaciones. Los tres ejemplos más paradigmáticos serían como hemos comentado, el método de generalización de factores aditivos, el análisis componencial y el análisis factorial confirmatorio. Estas estrategias se analizarán en un posterior artículo.

\section{Notas}

(1) El término coeficiente de cor-relación fue acuñado por el economista británico F. Y. Edgeworth (1845-1926).

(2) Según Harold Brown (1977, p. 144) «son las anomalías recalcitrantes las que llevan finalmente al derrocamiento de una teoría, y su sustitución por otra, es decir, a las revoluciones científicas".

(3) El título original "Comparative Studies of How Pepople Think» ha sido traducido al castellano, a nuestro parecer erróneamente, por «Cognición y Pensamiento: cómo pensamos, estudios comparados". 


\section{Referencias}

ANDERSON, J. R. (1976). Languaje, memory and thought. Hillsdale, N. J.: LEA.

BARON, J., y Treiman, R. (1980). Some problems in the study of cognitive processes. Memory and cognition, 8 (4), 313-321.

BerTalanFFY, L. (1974). Teoria general de sistemas. México: Fondo de Cultura Económica.

BODDY, J. (1978). Brain systems and psychological concepts. Chicester: John Willey and Sons.

BOWERS, K. E. (1973). Situationism psychology: an analysis and critic. Psychological Review, $80,307-337$.

BROWN, A. L. (1975). The development of memory: knowing about knowing, and knowing how to know. En H.W. Reese (comp.): Advances in child development and bebavior. Vol. 10. Nueva.York: Academic Press.

BRown, H. (1977). La nueva filosofia de la ciencia. Madrid: Tecnos [1983].

CALFEE, R. C. (1976). Sources of dependency on cognitive processes. En D. Klahr (comp.): Cognition and Instruction. Hislldale, N. J.: LEA.

CAMPIONE, J., y BROWN, A. (1979). Towards a theory of intelligence: contributions from the study with retarded children. En R. J. Sternberg y D. K. Detterman (comps.): Human intelligence. Norwood, N.J.: Ablex Publishing Corporation.

CAMpione, J.; Brown, A., y Ferrara, R. (1982). Mental retardation and intelligence. En R. J. Sternberg (comp.): Handbook of buman intelligence. Cambridge: Cambridge University Press.

CAPARros, A. (1982). Psicología diferencial: ¡ciencia o tecnología? Estudios de Psicología, 9, 15-23.

CARROLL, J. B. (1976). Psychometric tests as cognitive tasks: a new structure of intellect. En L. B. Resnick (comp.): The nature of intelligence. Hillsdale, N.J.: LEA.

CARroll, J. B. (1979). How shall we study individual differences in cognitive abilities? En R. J. Sternberg y D. K. Detterman (comps.): Human intelligence. Norwood, N.J.: Ablex Publishing Corporation.

CARROLL, J. B. (1981). Ability and task difficulty in cognitive psychology. Educational Researcher, 10, 11-21.

CARROLL, J. B. (1983). Studying individual differences in cognitive abilities: through and beyon factor analysis. En R. F. Dillon y R. R. Schmeck (comps.): Individual differences in cognition. Nueva York: Academic Press.

CATTELL, R. B. (1952). The thre basic factor-analytic research designs: their interrelations and derivatives. Psychological Bulletin, 49, 499-520.

Cattell, R. B. (1957). A universal index for psychological factors. Psychologia, 1, 74-85.

CLARK, H., y CHASE, W. (1972). On the process of comparing sentences against pictures. Cognitive Psychology, 3, 472-517.

COHEN, R. L., y SANDENBERG, T. (1977). Relation between intelligence and short term memory. Cognitive Psychology, 9, 534-554.

COLE, M., y MEANS, B. (1981). Comparative studies of how people think. An introduction. Cambridge, Mass. and Londres, Inglaterra: Harvard University Press. [Traducción española: Cognición y pensamiento. Cómo pensamos. Estudios comparados. Barcelona: Paidós, 1986].

CRONBACH, E. L. (1957). The two disciplines of scientific psychology. American Psychologist, 12, 671-684.

CronBACH, E. L. (1975). Beyond the two disciplines of scientific psychology. American Psychologist, 30, 116-127.

ChALKE, F., y ERTL, J. (1965). Evoked potentials and intelligence. Life Sciences, 4, 1319-1322.

Chapman, L. J., y Chapman, J. P. (1973). Problems in the measurement of cognitive deficit. Psychological Bulletin, 79, 380-385.

Chapman, L. J., y Chapman, J. P. (1985). Methodological problems in the study of differential deficits in regarded groups. En D. K. Detterman (comp.): Current topics in buman intelligence. Vol. 1: Research Metbodology. Norwood, N.J.: Ablex Publishing Corporation.

DHEN, N., y SCHANCK, R. (1982). Artificial and human intelligence. En R. J. Sternberg (comp.): Handbook of human intelligence. Cambridge: Cambridge University Press.

ELSHOUT, J. J. (1978). The characteristics demands of intellectual problems. En J. M. Scandura (comp.): Problem solving. Nueva York: Academic Press.

EYSENCK, H. J.(1985). Revolution in the theory and measurement of intelligence. Evaluación Psicológica, 1 (1-2), 99-158.

EYSENCK, H. J., y TURNEAUX (1982). Some major components in general intelligence. En P. O. White (comp.): A model for intelligence. Nueva York: Springer.

FARRELL, B. A. (1983). The correlation between behavior, body and mind. En A. Gale y J. A. Edwards (comps.): Physiological correlates of human behavior. Vol. I, Londres: Academic Press.

FeIGL, H. (1970). The Orthodox View of Theories. En M. Radner y S. Winokur (comps.): 
Minnesota Studies in the Pbilosophy of Sciences, IV. Mineapolis: University of Minnesota Press.

FleisCHMAN, E. A. (1967). Individual Differences and Motor Learning. En R. Gagne (comp.): Learning and Individual Differences. Columbus, Ohio: Charles E. Merril Publishing Company.

FODOR, J. A. (1968). La explicación psicológica. Madrid: Cátedra [1980].

FORTEZA y PRIETO (1981). Hacia una estructuración teórica de la psicología diferencial. Estudios de Psicologia, 4, 68-75.

FREDERIKSEN, J. R. (1978). Assessment of perceptual decoding and lexical skills, and their relation to reading proficiency. En A. M. Lesgolg, J. W. Pellegrino, S. Fokkeman y R. Glaser (comps.): Cognitive psychology and instruction. Nueva York: Plenum.

FRENCH, J. W. (1951). The description of aptitude and achievement tests in terms of rotated factors. Psychometric Monographs, 5 .

FRenCh, J. W.; EKstrom, R. B., y Price, L. A. (1963). Manual for kit of reference test for cognitive factors. Princeton, N.L.: Eductation Testing Service.

GAGNE, R. (1967). Learnin and individual differences. Columbus, Ohio: Charles E. Merril Publishing Company.

GALE, A., y EDWARDS, J. A. (1983). Introduction. En A. Gale y J. A. Edwards (comps.): Physiological correlates of human behavior. Vol. I. Londres: Academic Press.

GREGG, L. W. (1967). Simulation modelos and human differences. En R. Gagne (comp.): Learning and Individual Differences. Columbus, Ohio: Charles E. Merril Publishing Company.

GUILFORD, J. P. (1967). The nature of human intelligence. Londres: McGraw-Hill, International Studen Edition.

GUTOWSKI, W. E., y CHECILE, R. A. (1987). Encoding, storage and retrieval components of associative memory deficits of mildly mentally retarded adults. American Journal of Mental Deficienci, 92 (1), 85-93.

HeNDRICKSON, D. E. (1982). The biological basis of intelligence. Part II: Measurement. En H. J. Eysenck (comp.): A model for intelligence. Nueva York: Springer Verlag.

HUNT, E. (1971). What kind of computer is man? Cognitive Psychology, 2, 57-98.

HUNT, E.; Frost, N., y LUNNEBORG, C. (1973). Individual differences in cognition: a new approach to intelligence. En G. Bower (comp.): The psychology of learning and motivation. Vol. VII. Nueva York: Academic Press.

HunT, E., y LANSMAN, M. (1975). Cognitive theory applied to individual differences. En W. K. Estes (comp.): Handbook of learning and cognitive processes. Vol. 1. Hillsdale, N.J.: LEA.

HUNT, E., y MACLEOD, C. M. (1979). The sentence-verification paradigm: a case study of two conflicting approaches to individual differences. En R. J. Sternberg, y D. K. Detterman (comps.): Human intelligence. Norwood, N.J.: Ablex Publishing Corporation.

HuNT, E. (1985). The correlates of intelligence. En D. K. Detterman (comp.): Current topics in buman intelligence. Vol. 1 : Research methodology. Norwood, N.J.: Ablex Publishing Corporation.

Johnson, P. E.; Duran, A. S.; Hassebrock, F.; Moller, J.; Prietula, M.; Feltovich, P. J., y Swanson, D. B. (1981). Expertise and error in diagnostic reasoning. Cognitive Sciences, S, 235-284.

KJELDERGAARD, P. M. (1967). Simulation of cognition and learning: the role of individual differences. En R. Cagne (comp.): Learning and individual differences. Columbus, Ohio: Charles E. Merril Publishing Company.

KossLYN, S. M. (1980). Image and mind. Cambridge, Mass: Harward University Press.

KOSSLYN, S. M. (1984). A theory based approach to the study of individual differences in mental imagery. En R. E. Snow, R. A. Federico, y W. E. Montague (comps.): Aptitude, learning and instructions. Hillsdale, N.J.: LEA.

Kosslyn, S.; BRUNN, J; CAVE, K. R., y WALLACH, R. W. (1984). Individual differences in mental imagery ability: a computational analysis. Cognition, 18, 195-243.

KOTOVSKY, K., y SIMON, H. A. (1973). Empirical test of a theory of human acquisition of concepts for sequential patterns. Cognitive Psychology, 4, 399-424.

LAUGHERY, K. R., Y GREGG, L. W. (1962). The simulation of human problem solving behavior. Psychometrika, 27, 265-282.

LofTUS, G. R. (1978). On interpretation of interactions. Memory and Cognition, 6, 312-319.

LUNNEBORG, C. (1977). Choice reaction time: what role in ability measurement? Applied Psychological Measurement, $1,309-330$.

MARTINEz ARIAS, M. R. (1982). Inteligencia y procesamiento de la información. En I. Delclaux, y J. Seoane (comps.): Psicología cognitiva y procesamiento de la información. Madrid: Pirámide.

Mumaw, R. J.; Pellegrino, J. W., y Glaser, R. (1980). Some puzzling aspects of spatial ability. Comunicación presentada en la reunión anual de la Psychonomic Society, St. Louis.

Newell, A., y Simon, H. A. (1972). Human Problem Solving. Englewood Cliffs, N.J.: Prentice-Hall. 
Pellegrino, J. W., y Glaser, R. (1979). Cognitive correlates and components in the analysis of individual differences. Intelligence, 3, 187-214.

Pellegrino, J. W., y Glaser, R. (1980). Components of inductive reasoning. En R. E. Snow, P. A. Federico, y W. E. Montague (comps.): Aptitude, learning and instruction. Vol. 1: Cognitive processes of aptitude. Hillsdale, N.J.: LEA.

Pinillos, J. L. (1981). Observaciones sobre la psicología científica. En V. Pelechano, J. L. Pinillos, y J. Seoane (comps.): Psicologema. Valencia: Alfaplus, 27-76.

Peterson, L. R., y Peterson, M. J. (1959). Short term relation of individual verbal items. Journal of experimental Psychology, 58 (3), 515-524.

POSNER, M. I.; BOIES, S. I.; EICHELMAN, W. H., y TAYLOR, R. L. (1969). Retention of visual and name codes of single letters. Journal of Experimental Psychology Monographs, 79.

RoBINSON, D. L. (1982). Properties of diffuse thalamocortical system and human personality: a direct test of Pavlovian/Eysenckian theory. Personality and Individual Differences, 3, $1-16$.

ROSE, A. M. (1980). Information-processing abilities. En R. E. Snow, P. A. Federico, y W. E. Montague (comps.): Aptitude, learning and instruction. Vol. 1: Cognitive processes analysis of aptitude. Hillsdale, N.J.: LEA.

ROHWER, W. D. (1976). An introduction to research on individual and developmental differences in learning. En W. K. Estes (comp.): Handbook of learning and cognitive processes. Vol. 3. Hillsdale, N. J.: LEA.

ROYCE, J. (1973). The conceptual framework for a multifactor theory of individuality. En J. R. Royce (comp.): Multivariate analysis and psychological theory. Londres: Academic Press.

Russell Hanson, N. (1958). Patterns of discovery. [En castellano: Patrones de descubrimiento. Madrid: Alianza, 1977].

Sahakian, W. S. (1975). Historia y sistemas de la psicología. Madrid: Tecnos [1982].

SARGENT, S. S. (1942). How shall we study individual differences? Psychological Review, 49, $170-182$.

SEOANE, J. (1981). Problemas epistemológicos en la psicología actual. En V. Pelechano, J. L. Pinillos y J. Seoane (comps.): Psicologema. Valencia: Alfaplus, 27-76.

SEARLE, J. (1985). Cerebros, mentes y máquinas. Madrid: Cátedra [1986]

SCHAFER, E. W. B. (1982). Neural adaptability: a biological determinant of behavioral intelligence. International Journal of Neurosciences, 17, 183-191.

SEIGLER, R. S. (1976). Three aspects of cognitive development. Cognitive Psychology, 8, 481-520.

SimON, H. A., y KOTOvsKi, K. (1963). Human acquisiton of concepts for sequential patterns. Psychological Review, 70, 534-546.

SimoN, H. A. (1976). Identifying basic abilietes underlying intelligent performance of conplex task. En L. B. Resnick (comp.): The nature of intelligence. Hillsdale, N.J.: LEA.

SNOw, R. E. (1980). Aptitude processes. En R. E. Snow, P. A. Federico y W. E. Montague (comps.): Aptitude, learning and instruction. Vol. 1: Cognitive processes analysis of aptitude. Hillsdale, N.J.: LEA.

Stankov, L. (1980). Psychometric factors as cognitive task: a note on Carrol's new structure of intelect. Intelligence, 4, 65-71.

STERNBERG, S. (1969). Memory-scanning: mental processes revealed by reaction time experiments. American Scientist 57, 4, 421-457.

STERNBERG, R. J. (1977). Intelligence, information processing and analogical reasoning. The componential analysis of buman abilities. Hillsdale, N.J.: LEA.

Sternberg, R. J. (1981). Testing and cognitive psychology. American Psychologist, 36 (10), 1181-1189.

STERNBERG, R. J. (1985). Cognitive approaches to intelligence. En B. B. Wolman (comp.): Handbook of intelligence. Theories, measurements and applications. Nueva York: John Wiley.

TAYLOR, Ch. (1979). The explanation of purposive behavior. En Borger y Cioffi (comps.): Explanation in the behavioral sciences. Cambridge: Cambridge University Press.

Tous, J. M. (1986). Psicología de la personalidad: diferencias individuales, biológicas y cognitivas en el procesamiento de la información. Barcelona: PPU.

TZENG, O. J. L., y HUNG, D. L. (1985). From hemispheric to perceptual asymmetry: from where has all the variance come? En R. F. Dillon (comp.): Individual differences in cognition. Vol. 2. Nueva York: Academic Press.

VEGA, M. (1984). Introducción a la psicologia cognitiva. Madrid: Editorial Alianza.

YOUNG, R. M. (1978). Strategies and the structure of a cognitive skill. En G. Underwood (comp.): Strategies of information processing. Londres: Academic Press.

ZaCCAGNINI, J. L. (1985). Modelos de mundo como marcos de referencia a una psicología del conocimiento. Estudios de Psicología, 22. 